\title{
A SIMPLE OPTIMISED SEARCH HEURISTIC FOR THE JOB-SHOP SCHEDULING PROBLEM
}

\author{
Susana Fernandes \\ Universidade do Algarve, Faro, Portugal. \\ E-mail: sfer@ualg.pt \\ Helena R. Lourenço \\ Univertitat Pompeu Fabra, Barcelona, Spain. \\ E-mail: helena.ramalhinho@upf.edu
}

\begin{abstract}
This paper presents a simple Optimised Search Heuristic for the Job Shop Scheduling problem that combines a GRASP heuristic with a branch-and-bound algorithm. The proposed method is compared with similar approaches and leads to better results in terms of solution quality and computing times.
\end{abstract}

Keywords: job-shop scheduling, hybrid metaheuristic, optimised search heuristics, GRASP, exact methods.

JEL Codes: C61, M11

\section{Introduction}

The job shop scheduling problem has been known to the operations research community since the early 50's (Jain and Meeran 1999). It is considered a particularly hard combinatorial optimisation problem of the NP-hard class (Garey and Johnson 1979) and it has numerous practical applications; which makes it an excellent test problem for the quality of new scheduling algorithms. These are main reasons for the vast bibliography on both exact and heuristic methods applied to this particular scheduling problem. The paper of Jain and Meeran (1999) includes an exhaustive survey not only of the evolution of the definition of the problem, but also of all the techniques applied to it.

Recently a new class of procedures that combine local search based (meta) heuristics and exact algorithms have been developed, we denominate them Optimised Search Heuristics (OSH) (Fernandes and Lourenço 2007).

This paper presents a simple OSH procedure for the job shop scheduling problem that combines a GRASP heuristic with a branch-and-bound algorithm.

In the next section, we introduce the job shop scheduling problem. In section 2, we present a short review of existent OSH methods applied to this problem and in 
section 3 we describe in detail the OSH method developed. In section 5, we present the computational results along with comparisons to other similar procedures applied to the JSS problem. Section 6 concludes this paper and discusses some ideas for future research.

\section{The job shop scheduling problem}

The Job-Shop Scheduling Problem (JSSP) considers a set of jobs to be processed on a set of machines. Each job is defined by an ordered set of operations and each operation is assigned to a machine with a predefined constant processing time (preemption is not allowed). The order of the operations within the jobs and its correspondent machines are fixed a priori and independent from job to job. To solve the problem we need to find a sequence of operations on each machine respecting some constraints and optimising some objective function. It is assumed that two consecutive operations of the same job are assigned to different machines, each machine can only process one operation at a time and that different machines can not process the same job simultaneously. We will adopt the maximum of the completion time of all jobs - the makespan - as the objective function.

Formally let $O=\{0, \ldots, o+1\}$ be the set of operations with 0 and $0+1$ dummy operations representing the start and end of all jobs, respectively. Let $M$ be the set of machines, $A$ the set of arcs between consecutive operations of each job and $E_{k}$ the set of all possible pairs of operations processed by machine $\mathrm{k}$, with $k \in M$. We define $p_{i}>0$ as the constant processing time of operation $i$ and $t_{i}$ is the decision variable representing the start time of operation $i$. The following mathematical formulation for the job shop scheduling problem is widely used by researchers:

s.t.

$$
\begin{array}{ll}
\min t_{o+1} & \\
t_{j}-t_{i} \geq p_{i} & (i, j) \in A \\
t_{i} \geq 0 & i \in O \\
t_{j}-t_{i} \geq p_{i} \vee t_{i}-t_{j} \geq p_{j} & (i, j) \in E_{k}, k \in M
\end{array}
$$

The constraints in (1) state the precedence of operations within jobs and also that no two operations of the same job can be processed simultaneously (because $p_{i}>0$ ). 
Expressions (3) are named "capacity constraints” and assure there are no overlaps of operations at the machines. A feasible solution for the problem is a schedule of operations respecting all these constraints.

The job shop scheduling problem is usually represented by a disjunctive graph (Roy and Sussman 1964) $G=(O, A, E)$. Where $O$ is the node set, corresponding to the set of operations. $A$ is the set of arcs between consecutive operations of the same job, and $E$ is the set of edges between operations processed by the same machine. Each node $i$ has weight $p_{i}$, with $p_{0}=p_{o+1}=0$. There is a subset of nodes $O_{k}$ and a subset of edges $E_{k}$ for each machine that together form the disjunctive clique $C_{k}=\left(O_{k}, E_{k}\right)$ of graph $G$. For every node $j$ of $O /\{0, o+1\}$ there are unique nodes $i$ and $l$ such that $\operatorname{arcs}(i, j)$ and $(j, l)$ are elements of $A$. Node $i$ is called the job predecessor of node $j-j p(j)$ and $l$ is the job successor of $j-j s(j)$.

Finding a solution to the job shop scheduling problem means replacing every edge of the respective graph with a directed arc, constructing an acyclic directed graph $D_{S}=(O, A \cup S)$ where $S=\bigcup_{k} S_{k}$ corresponds to an acyclic union of sequences of operations for each machine $\mathrm{k}$ (this implies that a solution can be built sequencing one machine at a time). For any given solution, the operation processed immediately before operation $i$ in the same machine is called the machine predecessor of $i$ $m p(i)$; analogously $m s(i)$ is the operation that immediately succeeds $i$ at the same machine.

The optimal solution is the one represented by the graph $D_{S}$ having the critical path from 0 to $o+1$ with the smallest length.

\section{Review of Optimised Search Heuristics}

In the literature we can find a few works combining metaheuristics with exact algorithms applied to the job shop scheduling problem, designated as Optimized Search Heuristics (OSH) by Fernandes and Lourenço (2007). Different combinations of different procedures are present in the literature, and there are several applications of the OSH methods to different problems (see the web page of Fernandes and Lourenço (2007)) $)^{1}$.

\footnotetext{
${ }^{1}$ http://www.econ.upf.edu/ ramalhin/OSHwebpage/index.html
} 
Chen et al. (1993) and Denzinger and Offermann (1999) design parallel algorithms that use asynchronous agents information to build solutions; some of these agents are genetic algorithms, others are branch-and-bound algorithms.

Tamura et al (1994) design a genetic algorithm where the fitness of each individual, whose chromosomes represent each variable of the integer programming formulation, is the bound obtained solving lagrangian relaxations.

The works of Adams et al. (1988), Applegate and Cook (1991), Caseau and Laburthe (1995) and Balas and Vazacopoulos (1998) all use an exact algorithm to solve a sub problem within a local search heuristic for the job shop scheduling. Caseau and Laburthe (1995) build a local search where the neighbourhood structure is defined by a subproblem that is exactly solved using constraint programming. Applegate and Cook (1991) develop the shuffle heuristic. At each step of the local search the processing orders of the jobs on a small number of machines is fixed, and a branch-and-bound algorithm completes the schedule. The shifting bottleneck heuristic, due to Adams Balas and Zawack (1988), is an iterated local search with a construction heuristic that uses a branch-and-bound to solve the subproblems of one machine with release and due dates. Balas and Vazacopoulos (1998) work with the shifting bottleneck heuristic and design a guided local search, over a tree search structure, that reconstructs partially destroyed solutions.

Lourenço (1995) and Lourenço and Zwijnenburg (1996) use branch-and-bound algorithms to strategically guide an iterated local search and a tabu search algorithm. The diversification of the search is achieved by applying a branch-and-bound method to solve a one-machine scheduling problem subproblem obtained from the incumbent solution.

In the work of Schaal Fadil Silti and Tolla (1999) an interior point method generates initial solutions of the linear relaxation. A genetic algorithm finds integer solutions. A cut is generated based on the integer solutions found and the interior point method is applied again to diversify the search. This procedure is defined for the generalized job shop problem.

The interesting work of Danna Rothberg and Le Pape (2005) "applies the spirit of metaheuristics” in an exact algorithm. Within each node of a branch-and-cut tree, the solution of the linear relaxation is used to define the neighbourhood of the current best feasible solution. The local search consists in solving the restricted MIP problem defined by the neighbourhood. 


\section{Optimised search heuristic - GRASP_B\&B}

We developed a simple Optimised Search Heuristic that combines a GRASP algorithm with a branch-and-bound method. Here the branch-and-bound is used within the GRASP to solve subproblems of one machine scheduling.

GRASP means “Greedy Randomised Adaptive Search Procedure”, (Feo and Resende 1995). It is an iterative process where each iteration consists of two steps: a randomised building step of a greedy nature and a local search step. At the building phase, a feasible solution is constructed joining one element at a time. Each element is evaluated by a greedy function and incorporated (or not) in a restricted candidate list (RCL) according to its evaluation. The element to join the solution is chosen randomly from the RCL.

Each time a new element is added to the partial solution, if it has already more than one element, the algorithm proceeds with the local search step. The current solution is updated by the local optimum and this process of two steps is repeated until the solution is complete.

Next, we describe the OSH method GRASP_B\&B developed to solve the JobShop Scheduling problem. The main spirit of this heuristic is combining a GRASP method with a branch-and-bound to efficiently solve the JSSP.

\subsection{The Building step}

In this section, we describe in detail the building step of the GRASP_B\&B heuristic. We define the sequence of operations at each machine as the elements to join the solution, and the makespan $\left(\max \left(t_{i}+p_{i}\right), i \in O_{k}, k \in M\right)$ as the greedy function to evaluate them. In order to build the restricted candidate list (RCL), we find the optimal solution and optimal makespan, $f\left(x_{k}\right)$, for the one machine problems corresponding to all machines not yet scheduled. We identify the best $(\underline{f})$ and worst $(\bar{f})$ optimal makespans over all machines considered. A machine $k$ is included in the RCL if $f\left(x_{k}\right) \geq \bar{f}-\alpha(\bar{f}-\underline{f})$, where $f\left(x_{k}\right)$ is the makespan of machine $k$ and $\alpha$ is a uniform random number in $(0,1)$. This semi-greedy randomised procedure is biased towards the machine with the higher makespan, the bottleneck machine, in the sense 
that machines with low values of makespan have less probability of being included in the restricted candidate list.

\section{SemiGreedy $(K)$}

(1)

$$
\begin{array}{ll}
\text { (1) } & \alpha:=\operatorname{Random}(0,1) \\
\text { (2) } & \bar{f}:=\max \left\{f\left(x_{k}\right), k \in K\right\} \\
\text { (3) } & \underline{f}:=\min \left\{f\left(x_{k}\right), k \in K\right\} \\
\text { (4) } & R C L=\{\}
\end{array}
$$$$
\text { (3) } \quad \underline{f}:=\min \left\{f\left(x_{k}\right), k \in K\right\}
$$

(5) $\quad$ foreach $k \in K$

(8) return RandomChoice( $R C L)$

The building step requires a procedure to solve the one-machine scheduling problem. To solve this problem we use the branch-and-bound algorithm of Carlier (1982). The objective function of the algorithm is to minimize the completion time of all jobs. This one machine scheduling problem considers that to each job $j$ it is associated the following values that are obtained from the current solution: the processing time $\left(p_{j}\right)$, a release date $\left(r_{j}\right)$ and an amount of time $\left(q_{j}\right)$ that the job stays in the system after being processed. Considering the job shop problem and its disjunctive graph representation, the release date of each operation $i-\left(r_{i}\right)$ is obtained as the longest path from the beginning to $i$, and its tail $\left(q_{i}\right)$ as the longest path from $i$ to the end, without the processing time of $i$.

The one-machine branch-and-bound procedure implemented work as follows. At each node of the branch-and-bound tree the upper bound is computed using the algorithm of Schrage (1970). This algorithm gives priority to higher values of the tails $\left(q_{j}\right)$ when scheduling released jobs. We break ties by preferring larger processing times. The computation of the lower bound is based on the critical path with more jobs of the solution found by the algorithm of Schrage (1970) and on a critical job, defined by some properties proved by Carlier (1982). The value of the solution with preemption is used to strengthen this lower bound. We introduce a slight modification, forcing the lower bound of a node never to be smaller than the one of its 
father in the tree. The algorithm of Carlier (1982) uses some proven properties of the one machine scheduling problem to define the branching strategy, and also to reduce the number of inspected nodes of the branch-and-bound tree. When applying the algorithm to problems with 50 or more jobs, we observed that a lot of time was spent inspecting nodes of the tree, after having already found the optimal solution. So, to reduce the computational times, we introduced a condition restricting the number of nodes of the tree: the algorithm is stopped if there have been inspected more then $n^{3}$ nodes after the last reduction of the difference between the upper and lower bound of the tree ( $n$ is the number of jobs). We designated this procedure as Carlier_B\&B(k), where $k$ is the machine considered to be optimized and output the optimal onemachine schedule and the respective optimal value.

The way the one-machine branch-and-bound procedure is used within the building step is described next. At the first iteration we consider the graph $D=(O, A)$ (without the edges connecting operations that share the same machine) to compute release dates and tails. Incorporating a new machine in the solution means adding to the graph the arcs representing the sequence of operations in that machine. In terms of the mathematical formulation, this means choosing one of the inequalities of the disjunctive constraints (3) correspondent to the machine. We then update the makespan of the partial solution and the release dates and tails of unscheduled operations using the same procedure as the one used in the algorithm of Taillard (1994). We designate this procedure as TAILLARD $(x)$ that computes the makespan of a partial solution $x$ for the JSSP.

\subsection{The Local Search step}

In order to build a simple local search procedure we need to design a neighbourhood structure (defined by moves between solutions), the way to inspect the neighbourhood of a given solution, and a procedure to evaluate the quality of each neighbour solution. It is said that a solution B is a neighbour of a solution A if we can achieve B by performing a neighbourhood defining move in A.

We use a neighbourhood structure very similar to the NB neighbourhood of Dell'Amico and Trubian (1993) and the one of Balas and Vazacopoulos (1998). To describe the moves that define this neighbourhood we use the notion of blocks of critical operations. A block of critical operations is a maximal ordered set of 
consecutive operations of a critical path (in the disjunctive graph that represents the solution), sharing the same machine. Let $L(i, j)$ denote the length of the critical path from node $i$ to node $j$. Borrowing the nomination of Balas and Vazacopoulos (1998) we speak of forward and backward moves over forward and backward critical pairs of operations.

Two operations $u$ and $v$ form a forward critical pair $(u, v)$ if:
a) they both belong to the same block;
b) $v$ is the last operation of the block;
c) operation $j s(v)$ also belongs to the same critical path;
d) the length of the critical path from $v$ to $o+1$ is not less than the length of the critical path from $j s(u)$ to $o+1(L(v, o+1) \geq L(j s(u), o+1))$.

Two operations $u$ and $v$ form a backward critical pair $(u, v)$ if:
a) they both belong to the same block;
b) $u$ is the first operation of the block;
c) operation $j p(u)$ also belongs to the same critical path;
d) the length of the critical path from 0 to $u$, including the processing time of $u$, is not less than the length of the critical path from 0 to $j p(v)$, including the processing time of $\left.j p(v)\left(L(0, u)+p_{u} \geq L(0, j p(v))+p_{j p(v)}\right)\right)$.

Conditions d) are included to guarantee that all moves lead to feasible solutions (Balas and Vazacopoulos 1998). A forward move is executed by moving operation $u$ to be processed immediately after operation $v$. A backward move is executed by moving operation $v$ to be processed immediately before operation $u$.

The neighbourhood considered in the GRASP_B\&B is slightly different from the one considered in Dell'Amico and Trubian (1993) and Balas and Vazacopoulos (1998) since it considers partial solutions obtained at each iteration of the GRASP_B\&B heuristic. Therefore the local search is applied to a partial solution where a subset of all machines is scheduled. This neighbourhood is designated by $N(x, M \backslash K)$, where $x$ is a partial solution, $M$ is the set of all machines and $K$ is the set of machines not yet scheduled in the building phase. When inspecting the neighbourhood $N(x, M \backslash K)$, we stop whenever we find a neighbour with a best evaluation value than the makespan of $x$. 
To evaluate the quality of a neighbour of a partial solution $x$, obtained by a move over a critical pair $(u, v)$, we need only to compute the length of all the longest paths through the operations that were between $u$ and $v$ in the critical path of solution $x$. This evaluation is computed using the same procedure as the one used in the algorithm of Taillard (1994), TAILLARD(x).

The local search phase consists in the two procedures described in pseudo-code below:

\section{$\operatorname{LocalSearch}(x, f(x), M \backslash K)$}

(1) $\quad s:=$ neighbour $(x, f(x), M \backslash K)$

(2) while $s \neq x$

(3) $\quad x:=s$

(4) $\quad s:=$ neighbour $(x, f(x), M \backslash K)$

(5) return $s$

\section{Neighbour $(x, f(x), M \backslash K)$}

(1) foreach $s \in N(x, M \backslash K)$

(2) $\quad f(s):=$ evaluation $($ move $(x \rightarrow s))$

(3) $\quad$ if $f(s)<f(x)$

(4) $\quad$ return $s$

(5) $\quad$ return $x$

\subsection{GRASP_B\&B}

In this section, we present the complete GRASP_B\&B implemented, that considers the two phases previously described. Let runs be the total number of runs, $M$ the set of machines of the instance and $f(x)$ the makespan of a solution $x$. The full GRASP_B\&B method can be generally described by the pseudo-code as follows:

\section{GRASP_B\&B (runs)}

(1) $\quad M:=\{1, \cdots, m\}$

(2) for $r=1$ to runs

(3) $\quad x:=\{\}$

(4) $K:=M$

(5) while $K \neq\{\}$ 
(6)

$$
\begin{gathered}
\text { foreach } k \in K \\
x_{k}:=\text { CARLIER_B\& } B(k) \\
k^{*}:=\operatorname{SEMIGREEDY(K)} \\
x:=x \cup x_{k^{*}} \\
f(x):=\text { TAILLARD }(x) \\
K:=K \backslash\left\{k^{*}\right\} \\
\text { if }|K|<|M|-1 \\
\quad x:=L O C A L S E A R C H(x, M \backslash K) \\
\text { if } x^{*} \text { not initialised or } f(x)<f^{*} \\
x^{*}:=x \\
f^{*}:=f(x) \\
\text { return } x^{*} \quad
\end{gathered}
$$

This metaheuristic has only one parameter to be defined: the number of runs to perform (line (2)). The step of line (8) is the only one using randomness. When applied to an instance with $m$ machines, in each run of the metaheuristic, the branchand-bound algorithm is called $m \times(m+1) / 2$ times (line (7)); the local search is executed $m-1$ times (lines (12) and (13)); the procedure semigreedy (line (8)) and the algorithm of Taillard (line (10)) are executed $m$ times.

\section{Computational results}

We have tested the algorithm GRASP_B\&B on the benchmark instances abz5-9 (Adams et al. 1988), ft6, ft10, ft20 (Fisher and Thompson 1963), la01-40 (Lawrence 1984), orb01-10 (Applegate and Cook 1991), swv01-20 (Storer et al. 1992), ta01-70 (Taillard 1993) and yn1-4 (Yamada and Nakano 1992).

The tables have the following structure: in each line it is presented the name of the instance, the number of jobs and the number of machines of the instance $(n * m)$, and the best lower and upper bound values (LB, UB) of the makespan. If the lower bound is omitted, the upper bound is optimal. We gathered the values of these bounds from the paper of Jain and Meeran (1999) and the papers of Nowicki and Smutnicki (1996; 2002 and 2005).

The algorithm has been run 100 times for each instance on a Pentium 4 CPU 2.80 $\mathrm{GHz}$ and coded in $\mathrm{C}$. The tables also present some statistical values concerning the 
makespan of the solutions found in the 100 runs, as well as the total time of all runs (ttime) and the time to the best solution found (btime), in seconds. The statistics of the makespan computed over the 100 runs are the minimum (min), the first quartile (Q1), the median (Q2), the third quartile (Q3) and the maximum (max). We chose this measures because they allow us to see how disperse are the values obtain by different runs, which give us an idea of the robustness of the algorithm. Within brackets, next to each value , is the correspondent percentage of relative error to the upperbound:

$$
R E_{U B}(x)=100 \% \times \frac{f(x)-U B}{U B}
$$

Whenever the values are not worse than the best known upper bound, we present them in bold. Although this is a very simple (and fast) algorithm, it happens in 23 of the 152 instances used in this study.

\begin{tabular}{|c|c|c|c|c|c|c|c|c|c|c|}
\hline name & $\mathbf{n}^{*} \mathbf{m}$ & LB & UB & $\min$ & Q1 & Q2 & Q3 & $\max$ & ttime (s) & btime(s) \\
\hline abz5 & $10 * 10$ & & 1234 & $\begin{array}{l}1258 \\
(1.94)\end{array}$ & $\begin{array}{c}1312 \\
(6.32)\end{array}$ & $\begin{array}{l}1332 \\
(7.94)\end{array}$ & $\begin{array}{c}1358 \\
(10.05)\end{array}$ & $\begin{array}{c}1460 \\
(18.31)\end{array}$ & 0.7650 & 0.0995 \\
\hline abz6 & $10 * 10$ & & 943 & $\begin{array}{c}952 \\
(0.95)\end{array}$ & $\begin{array}{l}978.75 \\
(3.79)\end{array}$ & $\begin{array}{c}997 \\
(5.73)\end{array}$ & $\begin{array}{l}1012.5 \\
(7.37)\end{array}$ & $\begin{array}{c}1078 \\
(14.32)\end{array}$ & 0.7660 & 0.3064 \\
\hline abz7 & $15 * 20$ & & 656 & $\begin{array}{c}725 \\
(10.52)\end{array}$ & $\begin{array}{l}750.75 \\
(14.44)\end{array}$ & $\begin{array}{c}763 \\
(16.31)\end{array}$ & $\begin{array}{c}781 \\
(19.05)\end{array}$ & $\begin{array}{c}810 \\
(23.48)\end{array}$ & 10.9070 & 3.4902 \\
\hline abz8 & $15 * 20$ & 647 & 669 & $\begin{array}{c}734 \\
(9.72)\end{array}$ & $\begin{array}{c}767 \\
(14.65)\end{array}$ & $\begin{array}{c}780 \\
(16.59)\end{array}$ & $\begin{array}{l}797.25 \\
(19.17)\end{array}$ & $\begin{array}{c}837 \\
(25.11)\end{array}$ & 10.5160 & 1.8929 \\
\hline abz9 & $15 * 20$ & 661 & 679 & $\begin{array}{c}754 \\
(11.05)\end{array}$ & $\begin{array}{c}782.5 \\
(15.24)\end{array}$ & $\begin{array}{c}792 \\
(16.64)\end{array}$ & $\begin{array}{c}809 \\
(19.15)\end{array}$ & $\begin{array}{c}874 \\
(28.72)\end{array}$ & 10.4690 & 1.3610 \\
\hline
\end{tabular}

\begin{tabular}{|c|c|cc|ccccc|cc|}
\hline name & n*m & LB & UB & min & Q1 & Q2 & Q3 & max & $\begin{array}{c}\text { ttime } \\
\text { (s) }\end{array}$ & $\begin{array}{c}\text { btime(s } \\
\text { ) }\end{array}$ \\
\hline $\mathrm{ft} 06$ & & & & & & & & & & \\
& & & 55 & 55 & 59 & 59 & 61 & 66 & 0.1400 & 0.1274 \\
& & & & $(0.00)$ & $(7.27)$ & $(7.27)$ & $(10.91)$ & $(20.00)$ & & \\
\hline $\mathrm{ft} 10$ & $10 * 10$ & & 930 & 970 & 1026.75 & 1046 & 1073.25 & 1144 & 1.0000 & 0.5800 \\
& & & & $(4.30)$ & $(10.40)$ & $(12.47)$ & $(15.40)$ & $(23.01)$ & & 0.0094 \\
\hline $\mathrm{ft} 20$ & $20 * 5$ & & 1165 & 1283 & 1304 & 1318 & 1365 & 1409 & 0.4690 & \\
& & & & $(10.13)$ & $(11.93)$ & $(13.13)$ & $(17.17)$ & $(20.94)$ & & \\
\hline
\end{tabular}

\begin{tabular}{|c|c|c|c|c|c|c|c|c|c|c|}
\hline name & $\mathrm{n}^{*} \mathrm{~m}$ & LB & UB & $\min$ & Q1 & Q2 & Q3 & $\max$ & ttime(s) & btime(s) \\
\hline la01 & $10 * 5$ & & 666 & $\begin{array}{c}666 \\
(0.00)\end{array}$ & $\begin{array}{c}666 \\
(0.00)\end{array}$ & $\begin{array}{c}666 \\
(0.00)\end{array}$ & $\begin{array}{c}666 \\
(0.00)\end{array}$ & $\begin{array}{c}694 \\
(4.20)\end{array}$ & 0.1720 & 0.0017 \\
\hline $\begin{array}{ll}\text { la02 } \\
\end{array}$ & $10 * 5$ & & 655 & $\begin{array}{c}667 \\
(1.83)\end{array}$ & $\begin{array}{c}712 \\
(8.70)\end{array}$ & $\begin{array}{c}722 \\
(10.23)\end{array}$ & $\begin{array}{c}722 \\
(10.23)\end{array}$ & $\begin{array}{c}835 \\
(27.48)\end{array}$ & 0.1560 & 0.0437 \\
\hline la03 & $10 * 5$ & & 597 & $\begin{array}{c}605 \\
(1.34)\end{array}$ & $\begin{array}{c}605 \\
(1.34)\end{array}$ & $\begin{array}{c}640 \\
(7.20)\end{array}$ & $\begin{array}{c}701 \\
(17.42)\end{array}$ & $\begin{array}{c}701 \\
(17.42)\end{array}$ & 0.2190 & 0.0066 \\
\hline la04 & $10 * 5$ & & 590 & 607 & 610 & 648 & 648 & 672 & 0.1710 & 0.0051 \\
\hline
\end{tabular}




\begin{tabular}{|c|c|c|c|c|c|c|c|c|c|}
\hline & & & (2.88) & (3.39) & (9.83) & (9.83) & (13.90) & & \\
\hline la05 & $10 * 5$ & 593 & $\begin{array}{c}593 \\
(0.00)\end{array}$ & $\begin{array}{c}593 \\
(0.00)\end{array}$ & $\begin{array}{c}593 \\
(0.00)\end{array}$ & $\begin{array}{c}593 \\
(0.00)\end{array}$ & $\begin{array}{c}593 \\
(0.00)\end{array}$ & 0.1100 & 0.0011 \\
\hline la06 & $15 * 5$ & 926 & $\begin{array}{c}926 \\
(0.00)\end{array}$ & $\begin{array}{c}926 \\
(0.00)\end{array}$ & $\begin{array}{c}926 \\
(0.00)\end{array}$ & $\begin{array}{c}926 \\
(0.00)\end{array}$ & $\begin{array}{c}926 \\
(0.00)\end{array}$ & 0.1710 & 0.0017 \\
\hline la07 & $15 * 5$ & 890 & $\begin{array}{c}890 \\
(0.00)\end{array}$ & $\begin{array}{c}890 \\
(0.00)\end{array}$ & $\begin{array}{c}890 \\
(0.00)\end{array}$ & $\begin{array}{c}890 \\
(0.00)\end{array}$ & $\begin{array}{c}936 \\
(5.17)\end{array}$ & 0.2030 & 0.0020 \\
\hline la08 & $15 * 5$ & 863 & $\begin{array}{c}863 \\
(0.00)\end{array}$ & $\begin{array}{c}863 \\
(0.00)\end{array}$ & $\begin{array}{c}880 \\
(1.97)\end{array}$ & $\begin{array}{c}921 \\
(6.72)\end{array}$ & $\begin{array}{c}976 \\
(13.09)\end{array}$ & 0.2970 & 0.0149 \\
\hline la09 & $15 * 5$ & 951 & $\begin{array}{c}951 \\
(0.00)\end{array}$ & $\begin{array}{c}951 \\
(0.00)\end{array}$ & $\begin{array}{c}951 \\
(0.00)\end{array}$ & $\begin{array}{c}951 \\
(0.00)\end{array}$ & $\begin{array}{c}953 \\
(0.21)\end{array}$ & 0.2810 & 0.0028 \\
\hline la10 & $15 * 5$ & 958 & $\begin{array}{c}958 \\
(0.00)\end{array}$ & $\begin{array}{c}958 \\
(0.00)\end{array}$ & $\begin{array}{c}958 \\
(0.00)\end{array}$ & $\begin{array}{c}958 \\
(0.00)\end{array}$ & $\begin{array}{c}958 \\
(0.00)\end{array}$ & 0.1410 & 0.0014 \\
\hline la11 & $20 * 5$ & 1222 & $\begin{array}{l}1222 \\
(0.00)\end{array}$ & $\begin{array}{l}1222 \\
(0.00)\end{array}$ & $\begin{array}{l}1222 \\
(0.00)\end{array}$ & $\begin{array}{l}1222 \\
(0.00)\end{array}$ & $\begin{array}{c}1284 \\
(5.07)\end{array}$ & 0.2660 & 0.0027 \\
\hline la12 & $20 * 5$ & 1039 & $\begin{array}{l}1039 \\
(0.00)\end{array}$ & $\begin{array}{c}1039 \\
(0.00)\end{array}$ & $\begin{array}{c}1039 \\
(0.00)\end{array}$ & $\begin{array}{c}1039 \\
(0.00)\end{array}$ & $\begin{array}{c}1039 \\
(0.00)\end{array}$ & 0.2650 & 0.0027 \\
\hline la13 & $20 * 5$ & 1150 & $\begin{array}{l}1150 \\
(0.00)\end{array}$ & $\begin{array}{l}1150 \\
(0.00)\end{array}$ & $\begin{array}{l}1150 \\
(0.00)\end{array}$ & $\begin{array}{c}1150 \\
(0.00)\end{array}$ & $\begin{array}{l}1223 \\
(6.35)\end{array}$ & 0.3750 & 0.0038 \\
\hline la14 & $20 * 5$ & 1292 & $\begin{array}{l}1292 \\
(0.00)\end{array}$ & $\begin{array}{l}1292 \\
(0.00)\end{array}$ & $\begin{array}{l}1292 \\
(0.00)\end{array}$ & $\begin{array}{l}1292 \\
(0.00)\end{array}$ & $\begin{array}{l}1292 \\
(0.00)\end{array}$ & 0.2180 & 0.0022 \\
\hline la15 & $20 * 5$ & 1207 & $\begin{array}{l}1207 \\
(0.00)\end{array}$ & $\begin{array}{l}1240 \\
(2.73)\end{array}$ & $\begin{array}{l}1295 \\
(7.29)\end{array}$ & $\begin{array}{l}1295 \\
(7.29)\end{array}$ & $\begin{array}{l}1295 \\
(7.29)\end{array}$ & 0.9060 & 0.0453 \\
\hline la16 & $10 * 10$ & 945 & $\begin{array}{l}1012 \\
(7.09)\end{array}$ & $\begin{array}{l}1038.5 \\
(9.89)\end{array}$ & $\begin{array}{c}1049 \\
(11.01)\end{array}$ & $\begin{array}{c}1060 \\
(12.17)\end{array}$ & $\begin{array}{c}1099 \\
(16.30)\end{array}$ & 0.7350 & 0.0221 \\
\hline la17 & $10 * 10$ & 784 & $\begin{array}{c}787 \\
(0.38)\end{array}$ & $\begin{array}{l}813.75 \\
(3.79)\end{array}$ & $\begin{array}{l}836.5 \\
(6.70)\end{array}$ & $\begin{array}{l}864.25 \\
(10.24)\end{array}$ & $\begin{array}{c}950 \\
(21.17)\end{array}$ & 0.7660 & 0.0843 \\
\hline la18 & $10 * 10$ & 848 & $\begin{array}{c}854 \\
(0.71)\end{array}$ & $\begin{array}{l}879.25 \\
(3.69)\end{array}$ & $\begin{array}{c}895 \\
(5.54)\end{array}$ & $\begin{array}{c}924 \\
(8.96)\end{array}$ & $\begin{array}{c}1042 \\
(22.88)\end{array}$ & 0.7500 & 0.3000 \\
\hline la19 & $10 * 10$ & 842 & $\begin{array}{c}861 \\
(2.26)\end{array}$ & $\begin{array}{l}893.75 \\
(6.15)\end{array}$ & $\begin{array}{c}917 \\
(8.91)\end{array}$ & $\begin{array}{c}940.5 \\
(11.70)\end{array}$ & $\begin{array}{c}1020 \\
(21.14)\end{array}$ & 0.9690 & 0.4554 \\
\hline la20 & $10 * 10$ & 902 & $\begin{array}{c}920 \\
(2.00)\end{array}$ & $\begin{array}{c}960 \\
(6.43)\end{array}$ & $\begin{array}{c}976 \\
(8.20)\end{array}$ & $\begin{array}{l}1011.5 \\
(12.14)\end{array}$ & $\begin{array}{c}1080 \\
(19.73)\end{array}$ & 0.8130 & 0.0813 \\
\hline la21 & $15 * 10$ & 1046 & $\begin{array}{c}1092 \\
(4.40)\end{array}$ & $\begin{array}{c}1154 \\
(10.33)\end{array}$ & $\begin{array}{l}1177.5 \\
(12.57)\end{array}$ & $\begin{array}{l}1210.25 \\
(15.70)\end{array}$ & $\begin{array}{c}1286 \\
(22.94)\end{array}$ & 2.0460 & 0.1023 \\
\hline la22 & $15 * 10$ & 927 & $\begin{array}{c}955 \\
(3.02)\end{array}$ & $\begin{array}{c}999 \\
(7.77)\end{array}$ & $\begin{array}{l}1029.5 \\
(11.06)\end{array}$ & $\begin{array}{l}1063.5 \\
(14.72)\end{array}$ & $\begin{array}{c}1192 \\
(28.59)\end{array}$ & 1.7970 & 0.9884 \\
\hline la23 & $15 * 10$ & 1032 & $\begin{array}{l}1049 \\
(1.65)\end{array}$ & $\begin{array}{c}1089.25 \\
(5.55)\end{array}$ & $\begin{array}{c}1111 \\
(7.66)\end{array}$ & $\begin{array}{c}1136 \\
(10.08)\end{array}$ & $\begin{array}{c}1268 \\
(22.87)\end{array}$ & 1.8900 & 1.7388 \\
\hline la24 & $15 * 10$ & 935 & $\begin{array}{c}971 \\
(3.85)\end{array}$ & $\begin{array}{c}1016 \\
(8.66)\end{array}$ & $\begin{array}{c}1030 \\
(10.16)\end{array}$ & $\begin{array}{l}1054.25 \\
(12.75)\end{array}$ & $\begin{array}{c}1104 \\
(18.07)\end{array}$ & 1.8440 & 0.6270 \\
\hline la25 & $15 * 10$ & 977 & $\begin{array}{c}1027 \\
(5.12)\end{array}$ & $\begin{array}{l}1082.75 \\
(10.82)\end{array}$ & $\begin{array}{c}1100 \\
(12.59)\end{array}$ & $\begin{array}{l}1122.25 \\
(14.87)\end{array}$ & $\begin{array}{c}1226 \\
(25.49)\end{array}$ & 1.7960 & 0.5388 \\
\hline la26 & $20 * 10$ & 1218 & $\begin{array}{c}1265 \\
(3.86)\end{array}$ & $\begin{array}{c}1321.75 \\
(8.52)\end{array}$ & $\begin{array}{c}1355 \\
(11.25)\end{array}$ & $\begin{array}{c}1376 \\
(12.97)\end{array}$ & $\begin{array}{c}1485 \\
(21.92)\end{array}$ & 3.3750 & 3.0375 \\
\hline la27 & $20 * 10$ & 1235 & $\begin{array}{c}1308 \\
(5.91)\end{array}$ & $\begin{array}{c}1375 \\
(11.34)\end{array}$ & $\begin{array}{c}1399 \\
(13.28)\end{array}$ & $\begin{array}{l}1431.25 \\
(15.89)\end{array}$ & $\begin{array}{c}1538 \\
(24.53)\end{array}$ & 3.5620 & 0.1781 \\
\hline la28 & $20 * 10$ & 1216 & $\begin{array}{c}1301 \\
(6.99)\end{array}$ & $\begin{array}{c}1360.75 \\
(11.90)\end{array}$ & $\begin{array}{c}1391 \\
(14.39)\end{array}$ & $\begin{array}{l}1413.25 \\
(16.22)\end{array}$ & $\begin{array}{c}1533 \\
(26.07)\end{array}$ & 3.0000 & 0.1500 \\
\hline
\end{tabular}




\begin{tabular}{|c|c|c|c|c|c|c|c|c|c|}
\hline la29 & $20 * 10$ & 1152 & $\begin{array}{l}1248 \\
(8.33)\end{array}$ & $\begin{array}{l}1312.75 \\
(13.95)\end{array}$ & $\begin{array}{c}1339 \\
(16.23)\end{array}$ & $\begin{array}{c}1379 \\
(19.70)\end{array}$ & $\begin{array}{c}1466 \\
(27.26)\end{array}$ & 3.2960 & 0.8570 \\
\hline la30 & $20 * 10$ & 1355 & $\begin{array}{l}1382 \\
(1.99)\end{array}$ & $\begin{array}{c}1432.75 \\
(5.74)\end{array}$ & $\begin{array}{l}1452.5 \\
(7.20)\end{array}$ & $\begin{array}{c}1477 \\
(9.00)\end{array}$ & $\begin{array}{c}1548 \\
(14.24)\end{array}$ & 3.3280 & 0.8653 \\
\hline la31 & $30 * 10$ & 1784 & $\begin{array}{l}1784 \\
(0.00)\end{array}$ & $\begin{array}{c}1806.75 \\
(1.28)\end{array}$ & $\begin{array}{l}1829.5 \\
(2.55)\end{array}$ & $\begin{array}{c}1866.25 \\
(4.61)\end{array}$ & $\begin{array}{c}2006 \\
(12.44)\end{array}$ & 7.0160 & 0.0702 \\
\hline la32 & $30 * 10$ & 1850 & $\begin{array}{l}1850 \\
(0.00)\end{array}$ & $\begin{array}{c}1868.75 \\
(1.01)\end{array}$ & $\begin{array}{l}1906 \\
(3.03)\end{array}$ & $\begin{array}{c}1931 \\
(4.38)\end{array}$ & $\begin{array}{c}2024 \\
(9.41)\end{array}$ & 6.2350 & 0.5612 \\
\hline la33 & $30 * 10$ & 1719 & $\begin{array}{l}1719 \\
(0.00)\end{array}$ & $\begin{array}{c}1729.75 \\
(0.63)\end{array}$ & $\begin{array}{l}1756.5 \\
(2.18)\end{array}$ & $\begin{array}{c}1797 \\
(4.54)\end{array}$ & $\begin{array}{c}1872 \\
(8.90)\end{array}$ & 7.9060 & 1.2650 \\
\hline la34 & $30 * 10$ & 1721 & $\begin{array}{l}1721 \\
(0.00)\end{array}$ & $\begin{array}{c}1787 \\
(3.83)\end{array}$ & $\begin{array}{l}1812 \\
(5.29)\end{array}$ & $\begin{array}{c}1845.25 \\
(7.22)\end{array}$ & $\begin{array}{c}2025 \\
(17.66)\end{array}$ & 8.2810 & 3.8093 \\
\hline la35 & $30 * 10$ & 1888 & $\begin{array}{l}1888 \\
(0.00)\end{array}$ & $\begin{array}{c}1901 \\
(0.69)\end{array}$ & $\begin{array}{c}1923 \\
(1.85)\end{array}$ & $\begin{array}{c}1978.25 \\
(4.78)\end{array}$ & $\begin{array}{c}2232 \\
(18.22)\end{array}$ & 5.6880 & 0.2844 \\
\hline la36 & $15 * 15$ & 1268 & $\begin{array}{c}1325 \\
(4.50)\end{array}$ & $\begin{array}{c}1375.75 \\
(8.50)\end{array}$ & $\begin{array}{l}1395.5 \\
(10.06)\end{array}$ & $\begin{array}{l}1423.25 \\
(12.24)\end{array}$ & $\begin{array}{c}1521 \\
(19.95)\end{array}$ & 4.2650 & 0.0853 \\
\hline la37 & $15 * 15$ & 1397 & $\begin{array}{c}1479 \\
(5.87)\end{array}$ & $\begin{array}{l}1538.75 \\
(10.15)\end{array}$ & $\begin{array}{l}1565.5 \\
(12.06)\end{array}$ & $\begin{array}{l}1597.25 \\
(14.33)\end{array}$ & $\begin{array}{c}1642 \\
(17.54)\end{array}$ & 4.7970 & 4.0295 \\
\hline la38 & $15 * 15$ & 1196 & $\begin{array}{c}1274 \\
(6.52)\end{array}$ & $\begin{array}{l}1354.75 \\
(13.27)\end{array}$ & $\begin{array}{l}1381.5 \\
(15.51)\end{array}$ & $\begin{array}{l}1397.75 \\
(16.87)\end{array}$ & $\begin{array}{c}1471 \\
(22.99)\end{array}$ & 5.1090 & 0.7153 \\
\hline la39 & $15 * 15$ & 1233 & $\begin{array}{c}1309 \\
(6.16)\end{array}$ & $\begin{array}{c}1352.75 \\
(9.71)\end{array}$ & $\begin{array}{c}1374 \\
(11.44)\end{array}$ & $\begin{array}{l}1404.25 \\
(13.89)\end{array}$ & $\begin{array}{c}1468 \\
(19.06)\end{array}$ & 4.4530 & 2.9835 \\
\hline la40 & $15 * 15$ & 1222 & $\begin{array}{l}1291 \\
(5.65)\end{array}$ & $\begin{array}{c}1347 \\
(10.23)\end{array}$ & $\begin{array}{c}1369 \\
(12.03)\end{array}$ & $\begin{array}{l}1398.5 \\
(14.44)\end{array}$ & $\begin{array}{c}1451 \\
(18.74)\end{array}$ & 5.3910 & 3.5581 \\
\hline
\end{tabular}

\begin{tabular}{|c|c|c|c|c|c|c|c|c|c|c|}
\hline name & $n * m$ & LB & UB & $\min$ & Q1 & Q2 & Q3 & $\max$ & ttime(s) & btime(s) \\
\hline orb01 & $10 * 10$ & & 1059 & $\begin{array}{l}1145 \\
(8.12)\end{array}$ & $\begin{array}{l}1181.75 \\
(11.59)\end{array}$ & $\begin{array}{c}1198 \\
(13.13)\end{array}$ & $\begin{array}{l}1219.25 \\
(15.13)\end{array}$ & $\begin{array}{c}1335 \\
(26.06)\end{array}$ & 0.9850 & 0.0296 \\
\hline orb02 & $10 * 10$ & & 888 & $\begin{array}{c}918 \\
(3.38)\end{array}$ & $\begin{array}{c}959.75 \\
(8.08)\end{array}$ & $\begin{array}{c}983 \\
(10.70)\end{array}$ & $\begin{array}{c}1013.25 \\
(14.10)\end{array}$ & $\begin{array}{c}1085 \\
(22.18)\end{array}$ & 0.9530 & 0.0953 \\
\hline orb03 & $10 * 10$ & & 1005 & $\begin{array}{l}1098 \\
(9.25)\end{array}$ & $\begin{array}{l}1135.5 \\
(12.99)\end{array}$ & $\begin{array}{l}1155.5 \\
(14.98)\end{array}$ & $\begin{array}{l}1184.25 \\
(17.84)\end{array}$ & $\begin{array}{c}1289 \\
(28.26)\end{array}$ & 1.0150 & 0.3350 \\
\hline orb04 & $10 * 10$ & & 1005 & $\begin{array}{c}1066 \\
(6.07)\end{array}$ & $\begin{array}{c}1120 \\
(11.44)\end{array}$ & $\begin{array}{l}1144.5 \\
(13.88)\end{array}$ & $\begin{array}{c}1183 \\
(17.71)\end{array}$ & $\begin{array}{c}1255 \\
(24.88)\end{array}$ & 1.1250 & 0.8213 \\
\hline orb05 & $10 * 10$ & & 887 & $\begin{array}{c}911 \\
(2.71)\end{array}$ & $\begin{array}{c}966.75 \\
(8.99)\end{array}$ & $\begin{array}{c}1001 \\
(12.85)\end{array}$ & $\begin{array}{l}1014.25 \\
(14.35)\end{array}$ & $\begin{array}{c}1117 \\
(25.93)\end{array}$ & 0.8750 & 0.1050 \\
\hline orb06 & $10 * 10$ & & 1010 & $\begin{array}{c}1050 \\
(3.96)\end{array}$ & $\begin{array}{c}1108 \\
(9.70)\end{array}$ & $\begin{array}{l}1134.5 \\
(12.33)\end{array}$ & $\begin{array}{c}1172 \\
(16.04)\end{array}$ & $\begin{array}{c}1282 \\
(26.93)\end{array}$ & 1.0460 & 0.4812 \\
\hline orb07 & $10 * 10$ & & 397 & $\begin{array}{c}414 \\
(4.28)\end{array}$ & $\begin{array}{l}436.5 \\
(9.95)\end{array}$ & $\begin{array}{c}448 \\
(12.85)\end{array}$ & $\begin{array}{c}455 \\
(14.61)\end{array}$ & $\begin{array}{c}503 \\
(26.70)\end{array}$ & 1.0630 & 0.2764 \\
\hline orb08 & $10 * 10$ & & 899 & $\begin{array}{c}945 \\
(5.12)\end{array}$ & $\begin{array}{c}975 \\
(8.45)\end{array}$ & $\begin{array}{c}999 \\
(11.12)\end{array}$ & $\begin{array}{l}1032.75 \\
(14.88)\end{array}$ & $\begin{array}{c}1125 \\
(25.14)\end{array}$ & 1.0310 & 0.3093 \\
\hline orb09 & $10 * 10$ & & 934 & $\begin{array}{c}978 \\
(4.71)\end{array}$ & $\begin{array}{c}1003.75 \\
(7.47)\end{array}$ & $\begin{array}{c}1021 \\
(9.31)\end{array}$ & $\begin{array}{l}1053.75 \\
(12.82)\end{array}$ & $\begin{array}{c}1177 \\
(26.02)\end{array}$ & 0.9060 & 0.2809 \\
\hline orb10 & $10 * 10$ & & 944 & $\begin{array}{c}991 \\
(4.98)\end{array}$ & $\begin{array}{c}1024.75 \\
(8.55)\end{array}$ & $\begin{array}{c}1040 \\
(10.17)\end{array}$ & $\begin{array}{c}1073 \\
(13.67)\end{array}$ & $\begin{array}{c}1232 \\
(30.51)\end{array}$ & 0.8430 & 0.2276 \\
\hline
\end{tabular}

\begin{tabular}{|c|c|c|c|c|c|c|c|c|c|}
\hline name & $n * m$ & LB & UB & $\min$ & Q1 & Q2 & Q3 & $\max$ & ttime (s) \\
\hline
\end{tabular}




\begin{tabular}{|c|c|c|c|c|c|c|c|c|c|c|}
\hline swv01 & $20 * 10$ & & 1407 & $\begin{array}{c}1605 \\
(14.07)\end{array}$ & $\begin{array}{c}1688 \\
(19.97)\end{array}$ & $\begin{array}{c}1762 \\
(25.23)\end{array}$ & $\begin{array}{c}1806.75 \\
(28.41)\end{array}$ & $\begin{array}{c}1900 \\
(35.04)\end{array}$ & 3.6720 & 3.6720 \\
\hline swv02 & $20 * 10$ & & 1475 & $\begin{array}{c}1601 \\
(8.54)\end{array}$ & $\begin{array}{c}1696 \\
(14.98)\end{array}$ & $\begin{array}{c}1729 \\
(17.22)\end{array}$ & $\begin{array}{l}1776.5 \\
(20.44)\end{array}$ & $\begin{array}{c}1940 \\
(31.53)\end{array}$ & 3.2650 & 0.2939 \\
\hline swv03 & $20 * 10$ & 1369 & 1398 & $\begin{array}{c}1582 \\
(13.16)\end{array}$ & $\begin{array}{l}1666.75 \\
(19.22)\end{array}$ & $\begin{array}{l}1704.5 \\
(21.92)\end{array}$ & $\begin{array}{l}1738.5 \\
(24.36)\end{array}$ & $\begin{array}{c}1964 \\
(40.49)\end{array}$ & 3.4850 & 1.4986 \\
\hline swv04 & $20 * 10$ & 1450 & 1483 & $\begin{array}{c}1655 \\
(11.60)\end{array}$ & $\begin{array}{l}1737.5 \\
(17.16)\end{array}$ & $\begin{array}{l}1772.5 \\
(19.52)\end{array}$ & $\begin{array}{l}1816.25 \\
(22.47)\end{array}$ & $\begin{array}{c}1949 \\
(31.42)\end{array}$ & 4.0000 & 2.7200 \\
\hline swv05 & $20 * 10$ & & 1424 & $\begin{array}{c}1587 \\
(11.45)\end{array}$ & $\begin{array}{c}1660.75 \\
(16.63)\end{array}$ & $\begin{array}{c}1690 \\
(18.68)\end{array}$ & $\begin{array}{l}1718.25 \\
(20.66)\end{array}$ & $\begin{array}{c}1829 \\
(28.44)\end{array}$ & 3.6720 & 3.5986 \\
\hline swv06 & $20 * 15$ & 1591 & 1678 & $\begin{array}{c}1895 \\
(12.93)\end{array}$ & $\begin{array}{c}1975 \\
(17.70)\end{array}$ & $\begin{array}{l}2012.5 \\
(19.93)\end{array}$ & $\begin{array}{l}2064.25 \\
(23.02)\end{array}$ & $\begin{array}{c}2240 \\
(33.49)\end{array}$ & 10.7810 & 8.0858 \\
\hline swv07 & $20 * 15$ & 1446 & 1620 & $\begin{array}{c}1833 \\
(13.15)\end{array}$ & $\begin{array}{l}1881.75 \\
(16.16)\end{array}$ & $\begin{array}{c}1921 \\
(18.58)\end{array}$ & $\begin{array}{l}1953.75 \\
(20.60)\end{array}$ & $\begin{array}{c}2076 \\
(28.15)\end{array}$ & 11.5470 & 2.3094 \\
\hline swv08 & $20 * 15$ & 1640 & 1763 & $\begin{array}{c}2001 \\
(13.50)\end{array}$ & $\begin{array}{c}2103.25 \\
(19.30)\end{array}$ & $\begin{array}{c}2150 \\
(21.95)\end{array}$ & $\begin{array}{c}2190 \\
(24.22)\end{array}$ & $\begin{array}{c}2318 \\
(31.48)\end{array}$ & 11.0310 & 9.0454 \\
\hline swv09 & $20 * 15$ & 1604 & 1663 & $\begin{array}{c}1877 \\
(12.87)\end{array}$ & $\begin{array}{l}1984.75 \\
(19.35)\end{array}$ & $\begin{array}{l}2017.5 \\
(21.32)\end{array}$ & $\begin{array}{c}2088 \\
(25.56)\end{array}$ & $\begin{array}{c}2197 \\
(32.11)\end{array}$ & 11.3900 & 10.0232 \\
\hline swv10 & $20 * 15$ & 1631 & 1767 & $\begin{array}{c}1978 \\
(11.94)\end{array}$ & $\begin{array}{l}2053.5 \\
(16.21)\end{array}$ & $\begin{array}{c}2102 \\
(18.96)\end{array}$ & $\begin{array}{c}2145 \\
(21.39)\end{array}$ & $\begin{array}{c}2288 \\
(29.49)\end{array}$ & 10.0630 & 4.4277 \\
\hline swv11 & $50 * 10$ & 2983 & 2991 & $\begin{array}{c}3366 \\
(12.54)\end{array}$ & $\begin{array}{l}3454.25 \\
(15.49)\end{array}$ & $\begin{array}{l}3498.5 \\
(16.97)\end{array}$ & $\begin{array}{c}3574 \\
(19.49)\end{array}$ & $\begin{array}{c}4047 \\
(35.31)\end{array}$ & 62.3590 & 8.7303 \\
\hline swv12 & $50 * 10$ & 2972 & 3003 & $\begin{array}{c}3422 \\
(13.95)\end{array}$ & $\begin{array}{c}3520.75 \\
(17.24)\end{array}$ & $\begin{array}{c}3569 \\
(18.85)\end{array}$ & $\begin{array}{c}3621 \\
(20.58)\end{array}$ & $\begin{array}{c}4196 \\
(39.73)\end{array}$ & 141.9220 & 5.6769 \\
\hline swv13 & $50 * 10$ & & 3104 & $\begin{array}{c}3527 \\
(13.63)\end{array}$ & $\begin{array}{r}3601.25 \\
(16.02)\end{array}$ & $\begin{array}{c}3654 \\
(17.72)\end{array}$ & $\begin{array}{r}3698.25 \\
(19.14)\end{array}$ & $\begin{array}{c}4143 \\
(33.47)\end{array}$ & 54.9840 & 20.3441 \\
\hline swv14 & $50 * 10$ & & 2968 & $\begin{array}{c}3295 \\
(11.02)\end{array}$ & $\begin{array}{l}3362.25 \\
(13.28)\end{array}$ & $\begin{array}{l}3402.5 \\
(14.64)\end{array}$ & $\begin{array}{l}3469.5 \\
(16.90)\end{array}$ & $\begin{array}{c}4052 \\
(36.52)\end{array}$ & 180.8440 & 159.1427 \\
\hline swv15 & $50 * 10$ & 2885 & 2904 & $\begin{array}{c}3329 \\
(14.63)\end{array}$ & $\begin{array}{l}3458.5 \\
(19.09)\end{array}$ & $\begin{array}{c}3565 \\
(22.76)\end{array}$ & $\begin{array}{l}3634.25 \\
(25.15)\end{array}$ & $\begin{array}{c}3994 \\
(37.53)\end{array}$ & 113.1720 & 73.5618 \\
\hline swv16 & $50 * 10$ & & 2924 & $\begin{array}{c}2924 \\
(0.00)\end{array}$ & $\begin{array}{c}2924 \\
(0.00)\end{array}$ & $\begin{array}{c}2924 \\
(0.00)\end{array}$ & $\begin{array}{c}2924 \\
(0.00)\end{array}$ & $\begin{array}{l}2962 \\
(1.30)\end{array}$ & 9.6720 & 0.0967 \\
\hline swv17 & $50 * 10$ & & 2794 & $\begin{array}{c}2794 \\
(0.00)\end{array}$ & $\begin{array}{c}2794 \\
(0.00)\end{array}$ & $\begin{array}{c}2798 \\
(0.14)\end{array}$ & $\begin{array}{c}2828 \\
(1.22)\end{array}$ & $\begin{array}{c}2949 \\
(5.55)\end{array}$ & 16.9690 & 0.6788 \\
\hline swv18 & $50 * 10$ & & 2852 & $\begin{array}{c}2852 \\
(0.00)\end{array}$ & $\begin{array}{l}2852 \\
(0.00)\end{array}$ & $\begin{array}{c}2852 \\
(0.00)\end{array}$ & $\begin{array}{c}2879 \\
(0.95)\end{array}$ & $\begin{array}{r}2985 \\
(4.66)\end{array}$ & 15.6090 & 0.1561 \\
\hline swv19 & $50 * 10$ & & 2843 & $\begin{array}{c}2843 \\
(0.00)\end{array}$ & $\begin{array}{c}2864 \\
(0.74)\end{array}$ & $\begin{array}{c}2904 \\
(2.15)\end{array}$ & $\begin{array}{l}2972.5 \\
(4.56)\end{array}$ & $\begin{array}{c}3168 \\
(11.43)\end{array}$ & 30.2650 & 2.1186 \\
\hline swv20 & $50 * 10$ & & 2823 & $\begin{array}{c}\mathbf{2 8 2 3} \\
(0.00)\end{array}$ & $\begin{array}{c}\mathbf{2 8 2 3} \\
(0.00)\end{array}$ & $\begin{array}{c}2846.5 \\
(0.83)\end{array}$ & $\begin{array}{c}2894.25 \\
(2.52)\end{array}$ & $\begin{array}{l}3045 \\
(7.86)\end{array}$ & 17.3900 & 0.8695 \\
\hline
\end{tabular}

\begin{tabular}{|c|c|cc|ccccc|cc|}
\hline name & $\mathbf{n} * \mathbf{m}$ & $\mathbf{L B}$ & $\mathbf{U B}$ & $\mathbf{m i n}$ & $\mathbf{Q 1}$ & $\mathbf{Q 2}$ & $\mathbf{Q 3}$ & $\mathbf{m a x}$ & ttime (s) & btime (s) \\
\hline yn1 & $20 * 20$ & 826 & 888 & 955 & 996.75 & 1010.5 & 1031.25 & 1084 & 23.4530 & 4.2215 \\
& & & & $(7.55)$ & $(12.25)$ & $(13.80)$ & $(16.13)$ & $(22.07)$ & & \\
\hline yn2 & $20 * 20$ & 861 & 909 & 987 & 1035.75 & 1047 & 1060 & 1133 & 25.3750 & 12.4338 \\
& & & & $(8.58)$ & $(13.94)$ & $(15.18)$ & $(16.61)$ & $(24.64)$ & & \\
\hline yn3 & $20 * 20$ & 827 & 893 & 996 & 1029.75 & 1049 & 1068.5 & 1111 & 25.3430 & 11.9112 \\
& & & & $(11.53)$ & $(15.31)$ & $(17.47)$ & $(19.65)$ & $(24.41)$ & & \\
\hline yn4 & $20 * 20$ & 918 & 968 & 1060 & 1117.75 & 1132 & 1158 & 1209 & 23.8900 & 20.0676 \\
\hline
\end{tabular}


(9.50) (15.47) (16.94) (19.63) (24.90)

\begin{tabular}{|c|c|c|c|c|c|c|c|c|c|c|}
\hline name & $\mathbf{N * m}$ & LB & UB & $\min$ & Q1 & Q2 & Q3 & $\max$ & ttime (s) & btime (s) \\
\hline ta01 & $15 * 15$ & & 1231 & $\begin{array}{c}1332 \\
(8.20)\end{array}$ & $\begin{array}{c}1387 \\
(12.67)\end{array}$ & $\begin{array}{c}1413 \\
(14.78)\end{array}$ & $\begin{array}{l}1438.25 \\
(16.84)\end{array}$ & $\begin{array}{c}1556 \\
(26.40)\end{array}$ & 5.5630 & 0.2782 \\
\hline ta02 & $15 * 15$ & & 1244 & $\begin{array}{c}1313 \\
(5.55)\end{array}$ & $\begin{array}{c}1368.75 \\
(10.03)\end{array}$ & $\begin{array}{l}1394.5 \\
(12.10)\end{array}$ & $\begin{array}{l}1426.75 \\
(14.69)\end{array}$ & $\begin{array}{c}1499 \\
(20.50)\end{array}$ & 5.5150 & 4.9635 \\
\hline ta03 & $15 * 15$ & & 1218 & $\begin{array}{c}1278 \\
(4.93)\end{array}$ & $\begin{array}{l}1346.75 \\
(10.57)\end{array}$ & $\begin{array}{c}1370 \\
(12.48)\end{array}$ & $\begin{array}{l}1403.25 \\
(15.21)\end{array}$ & $\begin{array}{c}1488 \\
(22.17)\end{array}$ & 5.4060 & 4.1086 \\
\hline ta04 & $15 * 15$ & & 1175 & $\begin{array}{c}1249 \\
(6.30)\end{array}$ & $\begin{array}{c}1309 \\
(11.40)\end{array}$ & $\begin{array}{l}1330.5 \\
(13.23)\end{array}$ & $\begin{array}{l}1360.25 \\
(15.77)\end{array}$ & $\begin{array}{c}1518 \\
(29.19)\end{array}$ & 6.1560 & 3.4474 \\
\hline ta05 & $15 * 15$ & & 1224 & $\begin{array}{c}1310 \\
(7.03)\end{array}$ & $\begin{array}{c}1369 \\
(11.85)\end{array}$ & $\begin{array}{l}1393.5 \\
(13.85)\end{array}$ & $\begin{array}{c}1432 \\
(16.99)\end{array}$ & $\begin{array}{c}1579 \\
(29.00)\end{array}$ & 5.8120 & 0.8718 \\
\hline ta06 & $15 * 15$ & & 1238 & $\begin{array}{c}1308 \\
(5.65)\end{array}$ & $\begin{array}{l}1362.75 \\
(10.08)\end{array}$ & $\begin{array}{c}1396 \\
(12.76)\end{array}$ & $\begin{array}{l}1422.5 \\
(14.90)\end{array}$ & $\begin{array}{c}1535 \\
(23.99)\end{array}$ & 5.9370 & 0.7718 \\
\hline ta07 & $15 * 15$ & & 1227 & $\begin{array}{c}1299 \\
(5.87)\end{array}$ & $\begin{array}{c}1342 \\
(9.37)\end{array}$ & $\begin{array}{c}1364 \\
(11.17)\end{array}$ & $\begin{array}{l}1390.25 \\
(13.30)\end{array}$ & $\begin{array}{c}1549 \\
(26.24)\end{array}$ & 5.1400 & 2.3130 \\
\hline ta08 & $15 * 15$ & & 1217 & $\begin{array}{c}1306 \\
(7.31)\end{array}$ & $\begin{array}{c}1371 \\
(12.65)\end{array}$ & $\begin{array}{l}1389.5 \\
(14.17)\end{array}$ & $\begin{array}{l}1414.25 \\
(16.21)\end{array}$ & $\begin{array}{c}1523 \\
(25.14)\end{array}$ & 5.9530 & 2.7979 \\
\hline ta09 & $15 * 15$ & & 1274 & $\begin{array}{c}1395 \\
(9.50)\end{array}$ & $\begin{array}{c}1438 \\
(12.87)\end{array}$ & $\begin{array}{c}1465 \\
(14.99)\end{array}$ & $\begin{array}{c}1491 \\
(17.03)\end{array}$ & $\begin{array}{c}1614 \\
(26.69)\end{array}$ & 6.1100 & 1.5886 \\
\hline ta10 & $15 * 15$ & & 1241 & $\begin{array}{c}1332 \\
(7.33)\end{array}$ & $\begin{array}{c}1387 \\
(11.76)\end{array}$ & $\begin{array}{c}1413 \\
(13.86)\end{array}$ & $\begin{array}{l}1438.25 \\
(15.89)\end{array}$ & $\begin{array}{c}1556 \\
(25.38)\end{array}$ & 5.5150 & 0.2758 \\
\hline ta11 & $20 * 15$ & 1323 & 1361 & $\begin{array}{c}1497 \\
(9.99)\end{array}$ & $\begin{array}{c}1571 \\
(15.43)\end{array}$ & $\begin{array}{l}1597.5 \\
(17.38)\end{array}$ & $\begin{array}{l}1626.25 \\
(19.49)\end{array}$ & $\begin{array}{c}1727 \\
(26.89)\end{array}$ & 11.1100 & 7.9992 \\
\hline ta12 & $20 * 15$ & 1351 & 1367 & $\begin{array}{c}1511 \\
(10.53)\end{array}$ & $\begin{array}{l}1576.75 \\
(15.34)\end{array}$ & $\begin{array}{l}1590.5 \\
(16.35)\end{array}$ & $\begin{array}{l}1623.25 \\
(18.75)\end{array}$ & $\begin{array}{c}1709 \\
(25.02)\end{array}$ & 11.7650 & 3.4119 \\
\hline ta13 & $20 * 15$ & 1282 & 1342 & $\begin{array}{c}1498 \\
(11.62)\end{array}$ & $\begin{array}{l}1559.5 \\
(16.21)\end{array}$ & $\begin{array}{l}1581.5 \\
(17.85)\end{array}$ & $\begin{array}{l}1618.25 \\
(20.58)\end{array}$ & $\begin{array}{c}1728 \\
(28.76)\end{array}$ & 10.6880 & 5.0234 \\
\hline ta14 & $20 * 15$ & & 1345 & $\begin{array}{c}1439 \\
(6.99)\end{array}$ & $\begin{array}{l}1496.75 \\
(11.28)\end{array}$ & $\begin{array}{l}1527.5 \\
(13.57)\end{array}$ & $\begin{array}{c}1569 \\
(16.65)\end{array}$ & $\begin{array}{c}1692 \\
(25.80)\end{array}$ & 11.5940 & 2.3188 \\
\hline ta15 & $20 * 15$ & 1304 & 1340 & $\begin{array}{c}1511 \\
(12.76)\end{array}$ & $\begin{array}{l}1576.25 \\
(17.63)\end{array}$ & $\begin{array}{c}1602 \\
(19.55)\end{array}$ & $\begin{array}{c}1639 \\
(22.31)\end{array}$ & $\begin{array}{c}1732 \\
(29.25)\end{array}$ & 12.4380 & 4.3533 \\
\hline ta16 & $20 * 15$ & 1302 & 1360 & $\begin{array}{c}1486 \\
(9.26)\end{array}$ & $\begin{array}{l}1551.75 \\
(14.10)\end{array}$ & $\begin{array}{l}1571.5 \\
(15.55)\end{array}$ & $\begin{array}{c}1609.25 \\
(18.33)\end{array}$ & $\begin{array}{c}1677 \\
(23.31)\end{array}$ & 11.2030 & 5.9376 \\
\hline ta17 & $20 * 15$ & & 1462 & $\begin{array}{c}1600 \\
(9.44)\end{array}$ & $\begin{array}{c}1661 \\
(13.61)\end{array}$ & $\begin{array}{l}1693.5 \\
(15.83)\end{array}$ & $\begin{array}{c}1713 \\
(17.17)\end{array}$ & $\begin{array}{c}1911 \\
(30.71)\end{array}$ & 9.3910 & 7.7006 \\
\hline ta18 & $20 * 15$ & 1369 & 1396 & $\begin{array}{c}1543 \\
(10.53)\end{array}$ & $\begin{array}{c}1623 \\
(16.26)\end{array}$ & $\begin{array}{c}1652 \\
(18.34)\end{array}$ & $\begin{array}{l}1677.25 \\
(20.15)\end{array}$ & $\begin{array}{c}1782 \\
(27.65)\end{array}$ & 12.2030 & 7.5659 \\
\hline ta19 & $20 * 15$ & 1297 & 1335 & $\begin{array}{c}1463 \\
(9.59)\end{array}$ & $\begin{array}{c}1542 \\
(15.51)\end{array}$ & $\begin{array}{c}1574 \\
(17.90)\end{array}$ & $\begin{array}{c}1616 \\
(21.05)\end{array}$ & $\begin{array}{c}1740 \\
(30.34)\end{array}$ & 11.5310 & 7.2645 \\
\hline ta20 & $20 * 15$ & 1318 & 1351 & $\begin{array}{c}1498 \\
(10.88)\end{array}$ & $\begin{array}{c}1549 \\
(14.66)\end{array}$ & $\begin{array}{c}1580 \\
(16.95)\end{array}$ & $\begin{array}{c}1617 \\
(19.69)\end{array}$ & $\begin{array}{c}1686 \\
(24.80)\end{array}$ & 11.5620 & 6.2435 \\
\hline ta21 & $20 * 20$ & 1539 & 1644 & $\begin{array}{c}1810 \\
(10.10)\end{array}$ & $\begin{array}{l}1894.5 \\
(15.24)\end{array}$ & $\begin{array}{c}1936 \\
(17.76)\end{array}$ & $\begin{array}{l}1970.25 \\
(19.84)\end{array}$ & $\begin{array}{c}2144 \\
(30.41)\end{array}$ & 20.9690 & 11.1136 \\
\hline ta22 & $20 * 20$ & 1511 & 1600 & $\begin{array}{c}1792 \\
(12.00)\end{array}$ & $\begin{array}{l}1832.75 \\
(14.55)\end{array}$ & $\begin{array}{c}1865 \\
(16.56)\end{array}$ & $\begin{array}{c}1903 \\
(18.94)\end{array}$ & $\begin{array}{c}1989 \\
(24.31)\end{array}$ & 22.4840 & 6.0707 \\
\hline ta23 & $20 * 20$ & 1472 & 1557 & $\begin{array}{c}1708 \\
(9.70)\end{array}$ & $\begin{array}{c}1768.75 \\
(13.60)\end{array}$ & $\begin{array}{c}1801 \\
(15.67)\end{array}$ & $\begin{array}{c}1839.25 \\
(18.13)\end{array}$ & $\begin{array}{c}1947 \\
(25.05)\end{array}$ & 22.0780 & 16.1169 \\
\hline
\end{tabular}




\begin{tabular}{|c|c|c|c|c|c|c|c|c|c|c|}
\hline ta24 & $20 * 20$ & 1602 & 1647 & $\begin{array}{l}1778 \\
(7.95)\end{array}$ & $\begin{array}{l}1864.75 \\
(13.22)\end{array}$ & $\begin{array}{l}1894.5 \\
(15.03)\end{array}$ & $\begin{array}{l}1925.25 \\
(16.89)\end{array}$ & $\begin{array}{c}2014 \\
(22.28)\end{array}$ & 19.1870 & 17.0764 \\
\hline ta25 & $20 * 20$ & 1504 & 1595 & $\begin{array}{l}1746 \\
(9.47)\end{array}$ & $\begin{array}{l}1830.75 \\
(14.78)\end{array}$ & $\begin{array}{c}1876 \\
(17.62)\end{array}$ & $\begin{array}{l}1913.5 \\
(19.97)\end{array}$ & $\begin{array}{c}1992 \\
(24.89)\end{array}$ & 20.4060 & 16.1207 \\
\hline ta26 & $20 * 20$ & 1539 & 1645 & $\begin{array}{l}1768 \\
(7.48)\end{array}$ & $\begin{array}{l}1863.75 \\
(13.30)\end{array}$ & $\begin{array}{c}1907 \\
(15.93)\end{array}$ & $\begin{array}{l}1950.25 \\
(18.56)\end{array}$ & $\begin{array}{c}2027 \\
(23.22)\end{array}$ & 17.8440 & 2.6766 \\
\hline ta27 & $20 * 20$ & 1616 & 1680 & $\begin{array}{l}1839 \\
(9.46)\end{array}$ & $\begin{array}{l}1923.75 \\
(14.51)\end{array}$ & $\begin{array}{c}1954 \\
(16.31)\end{array}$ & $\begin{array}{l}1988.25 \\
(18.35)\end{array}$ & $\begin{array}{c}2149 \\
(27.92)\end{array}$ & 19.8440 & 17.8596 \\
\hline ta28 & $20 * 20$ & 1591 & 1614 & $\begin{array}{l}1755 \\
(8.74)\end{array}$ & $\begin{array}{l}1837.5 \\
(13.85)\end{array}$ & $\begin{array}{c}1871 \\
(15.92)\end{array}$ & $\begin{array}{l}1908.75 \\
(18.26)\end{array}$ & $\begin{array}{c}2016 \\
(24.91)\end{array}$ & 22.3130 & 19.1892 \\
\hline ta29 & $20 * 20$ & 1514 & 1625 & $\begin{array}{l}1717 \\
(5.66)\end{array}$ & $\begin{array}{l}1835.75 \\
(12.97)\end{array}$ & $\begin{array}{c}1864 \\
(14.71)\end{array}$ & $\begin{array}{c}1898.25 \\
(16.82)\end{array}$ & $\begin{array}{c}2012 \\
(23.82)\end{array}$ & 20.1570 & 6.2487 \\
\hline ta30 & $20 * 20$ & 1473 & 1584 & $\begin{array}{c}1737 \\
(9.66)\end{array}$ & $\begin{array}{c}1800.75 \\
(13.68)\end{array}$ & $\begin{array}{l}1827.5 \\
(15.37)\end{array}$ & $\begin{array}{l}1852.5 \\
(16.95)\end{array}$ & $\begin{array}{c}1960 \\
(23.74)\end{array}$ & 17.5470 & 6.4924 \\
\hline ta31 & $30 * 15$ & & 1764 & $\begin{array}{c}1976 \\
(12.02)\end{array}$ & $\begin{array}{c}2059.75 \\
(16.77)\end{array}$ & $\begin{array}{l}2099.5 \\
(19.02)\end{array}$ & $\begin{array}{c}2135 \\
(21.03)\end{array}$ & $\begin{array}{c}2322 \\
(31.63)\end{array}$ & 30.6090 & 14.3862 \\
\hline ta32 & $30 * 15$ & 1774 & 1796 & $\begin{array}{c}2029 \\
(12.97)\end{array}$ & $\begin{array}{l}2132.5 \\
(18.74)\end{array}$ & $\begin{array}{l}2165.5 \\
(20.57)\end{array}$ & $\begin{array}{c}2205 \\
(22.77)\end{array}$ & $\begin{array}{c}2356 \\
(31.18)\end{array}$ & 30.6090 & 23.8750 \\
\hline ta33 & $30 * 15$ & 1778 & 1793 & $\begin{array}{c}2070 \\
(15.45)\end{array}$ & $\begin{array}{l}2171.25 \\
(21.10)\end{array}$ & $\begin{array}{c}2204 \\
(22.92)\end{array}$ & $\begin{array}{c}2265 \\
(26.32)\end{array}$ & $\begin{array}{c}2336 \\
(30.28)\end{array}$ & 31.9220 & 18.1955 \\
\hline ta34 & $30 * 15$ & 1828 & 1829 & $\begin{array}{c}2024 \\
(10.66)\end{array}$ & $\begin{array}{l}2114.25 \\
(15.60)\end{array}$ & $\begin{array}{c}2156 \\
(17.88)\end{array}$ & $\begin{array}{c}2186 \\
(19.52)\end{array}$ & $\begin{array}{c}2287 \\
(25.04)\end{array}$ & 33.6720 & 32.3251 \\
\hline ta35 & $30 * 15$ & & 2007 & $\begin{array}{l}2093 \\
(4.29)\end{array}$ & $\begin{array}{c}2174.25 \\
(8.33)\end{array}$ & $\begin{array}{c}2208 \\
(10.01)\end{array}$ & $\begin{array}{l}2250.25 \\
(12.12)\end{array}$ & $\begin{array}{c}2454 \\
(22.27)\end{array}$ & 35.2970 & 13.4129 \\
\hline ta36 & $30 * 15$ & & 1819 & $\begin{array}{c}2040 \\
(12.15)\end{array}$ & $\begin{array}{l}2124.5 \\
(16.79)\end{array}$ & $\begin{array}{l}2153.5 \\
(18.39)\end{array}$ & $\begin{array}{l}2204.25 \\
(21.18)\end{array}$ & $\begin{array}{c}2307 \\
(26.83)\end{array}$ & 30.5310 & 3.9690 \\
\hline ta37 & $30 * 15$ & 1771 & 1778 & $\begin{array}{c}1967 \\
(10.63)\end{array}$ & $\begin{array}{c}2099.75 \\
(18.10)\end{array}$ & $\begin{array}{c}2136 \\
(20.13)\end{array}$ & $\begin{array}{l}2184.5 \\
(22.86)\end{array}$ & $\begin{array}{c}2408 \\
(35.43)\end{array}$ & 29.0310 & 15.3864 \\
\hline ta38 & $30 * 15$ & & 1673 & $\begin{array}{c}1913 \\
(14.35)\end{array}$ & $\begin{array}{c}1976.75 \\
(18.16)\end{array}$ & $\begin{array}{l}2003.5 \\
(19.75)\end{array}$ & $\begin{array}{l}2046.25 \\
(22.31)\end{array}$ & $\begin{array}{c}2188 \\
(30.78)\end{array}$ & 34.0000 & 12.5800 \\
\hline ta39 & $30 * 15$ & & 1795 & $\begin{array}{l}1966 \\
(9.53)\end{array}$ & $\begin{array}{l}2069.25 \\
(15.28)\end{array}$ & $\begin{array}{l}2107.5 \\
(17.41)\end{array}$ & $\begin{array}{c}2144 \\
(19.44)\end{array}$ & $\begin{array}{c}2321 \\
(29.30)\end{array}$ & 31.3750 & 13.4913 \\
\hline ta40 & $30 * 15$ & 1631 & 1674 & $\begin{array}{c}1931 \\
(15.35)\end{array}$ & $\begin{array}{c}2012 \\
(20.19)\end{array}$ & $\begin{array}{l}2047.5 \\
(22.31)\end{array}$ & $\begin{array}{l}2077.25 \\
(24.09)\end{array}$ & $\begin{array}{c}2218 \\
(32.50)\end{array}$ & 33.9850 & 0.3399 \\
\hline ta41 & $30 * 20$ & 1859 & 2018 & $\begin{array}{c}2348 \\
(16.35)\end{array}$ & $\begin{array}{c}2413.75 \\
(19.61)\end{array}$ & $\begin{array}{c}2458 \\
(21.80)\end{array}$ & $\begin{array}{c}2494 \\
(23.59)\end{array}$ & $\begin{array}{c}2638 \\
(30.72)\end{array}$ & 61.1100 & 0.6111 \\
\hline ta42 & $30 * 20$ & 1867 & 1956 & $\begin{array}{c}2206 \\
(12.78)\end{array}$ & $\begin{array}{c}2301.75 \\
(17.68)\end{array}$ & $\begin{array}{c}2341 \\
(19.68)\end{array}$ & $\begin{array}{c}2374.25 \\
(21.38)\end{array}$ & $\begin{array}{c}2513 \\
(28.48)\end{array}$ & 62.3280 & 49.2391 \\
\hline ta43 & $30 * 20$ & 1809 & 1859 & $\begin{array}{c}2155 \\
(15.92)\end{array}$ & $\begin{array}{l}2228.5 \\
(19.88)\end{array}$ & $\begin{array}{c}2254 \\
(21.25)\end{array}$ & $\begin{array}{l}2294.5 \\
(23.43)\end{array}$ & $\begin{array}{c}2395 \\
(28.83)\end{array}$ & 72.7040 & 50.8928 \\
\hline ta44 & $30 * 20$ & 1927 & 1984 & $\begin{array}{c}2300 \\
(15.93)\end{array}$ & $\begin{array}{l}2382.5 \\
(20.09)\end{array}$ & $\begin{array}{c}2418 \\
(21.88)\end{array}$ & $\begin{array}{c}2466 \\
(24.29)\end{array}$ & $\begin{array}{c}2577 \\
(29.89)\end{array}$ & 64.7970 & 11.6635 \\
\hline ta45 & $30 * 20$ & 1997 & 2000 & $\begin{array}{c}2295 \\
(14.75)\end{array}$ & $\begin{array}{c}2358 \\
(17.90)\end{array}$ & $\begin{array}{c}2380 \\
(19.00)\end{array}$ & $\begin{array}{c}2410.25 \\
(20.51)\end{array}$ & $\begin{array}{c}2581 \\
(29.05)\end{array}$ & 70.8280 & 34.7057 \\
\hline ta46 & $30 * 20$ & 1940 & 2021 & $\begin{array}{c}2314 \\
(14.50)\end{array}$ & $\begin{array}{l}2399.5 \\
(18.73)\end{array}$ & $\begin{array}{c}2438 \\
(20.63)\end{array}$ & $\begin{array}{c}2481 \\
(22.76)\end{array}$ & $\begin{array}{c}2660 \\
(31.62)\end{array}$ & 64.1090 & 25.6436 \\
\hline ta47 & $30 * 20$ & 1789 & 1903 & $\begin{array}{c}2151 \\
(13.03)\end{array}$ & $\begin{array}{l}2260.5 \\
(18.79)\end{array}$ & $\begin{array}{l}2299.5 \\
(20.84)\end{array}$ & $\begin{array}{c}2345.75 \\
(23.27)\end{array}$ & $\begin{array}{c}2443 \\
(28.38)\end{array}$ & 63.9850 & 63.3452 \\
\hline ta48 & $30 * 20$ & 1912 & 1952 & 2222 & 2325.5 & 2360 & 2407.5 & 2565 & 63.2190 & 60.0581 \\
\hline
\end{tabular}




\begin{tabular}{|c|c|c|c|c|c|c|c|c|c|c|}
\hline & & & & (13.83) & (19.13) & (20.90) & (23.34) & (31.40) & & \\
\hline ta49 & $30 * 20$ & 1915 & 1968 & $\begin{array}{c}2250 \\
(14.33)\end{array}$ & $\begin{array}{l}2349.5 \\
(19.39)\end{array}$ & $\begin{array}{c}2390 \\
(21.44)\end{array}$ & $\begin{array}{c}2425 \\
(23.22)\end{array}$ & $\begin{array}{c}2560 \\
(30.08)\end{array}$ & 65.2650 & 11.7477 \\
\hline ta50 & $30 * 20$ & 1807 & 1928 & $\begin{array}{c}2264 \\
(17.43)\end{array}$ & $\begin{array}{l}2347.5 \\
(21.76)\end{array}$ & $\begin{array}{c}2387 \\
(23.81)\end{array}$ & $\begin{array}{c}2431 \\
(26.09)\end{array}$ & $\begin{array}{c}2633 \\
(36.57)\end{array}$ & 63.2970 & 13.2924 \\
\hline ta51 & $50 * 15$ & & 2760 & $\begin{array}{c}3001 \\
(8.73)\end{array}$ & $\begin{array}{c}3149 \\
(14.09)\end{array}$ & $\begin{array}{c}3227 \\
(16.92)\end{array}$ & $\begin{array}{c}3294 \\
(19.35)\end{array}$ & $\begin{array}{c}3587 \\
(29.96)\end{array}$ & 139.8750 & 65.7413 \\
\hline ta52 & $50 * 15$ & & 2756 & $\begin{array}{l}2940 \\
(6.68)\end{array}$ & $\begin{array}{c}3136 \\
(13.79)\end{array}$ & $\begin{array}{c}3216 \\
(16.69)\end{array}$ & $\begin{array}{l}3276.5 \\
(18.89)\end{array}$ & $\begin{array}{c}3590 \\
(30.26)\end{array}$ & 128.3590 & 119.3739 \\
\hline ta53 & $50 * 15$ & & 2717 & $\begin{array}{l}2895 \\
(6.55)\end{array}$ & $\begin{array}{c}2998 \\
(10.34)\end{array}$ & $\begin{array}{l}3031.5 \\
(11.58)\end{array}$ & $\begin{array}{l}3072.5 \\
(13.08)\end{array}$ & $\begin{array}{c}3219 \\
(18.48)\end{array}$ & 116.9370 & 87.7028 \\
\hline ta54 & $50 * 15$ & & 2839 & $\begin{array}{c}2914 \\
(2.64)\end{array}$ & $\begin{array}{c}3024.75 \\
(6.54)\end{array}$ & $\begin{array}{c}3092.5 \\
(8.93)\end{array}$ & $\begin{array}{l}3133.75 \\
(10.38)\end{array}$ & $\begin{array}{c}3280 \\
(15.53)\end{array}$ & 112.5470 & 15.7566 \\
\hline ta55 & $50 * 15$ & & 2679 & $\begin{array}{c}2988 \\
(11.53)\end{array}$ & $\begin{array}{c}3133 \\
(16.95)\end{array}$ & $\begin{array}{c}3178 \\
(18.63)\end{array}$ & $\begin{array}{l}3247.5 \\
(21.22)\end{array}$ & $\begin{array}{c}3440 \\
(28.41)\end{array}$ & 144.6570 & 56.4162 \\
\hline ta56 & $50 * 15$ & & 2781 & $\begin{array}{c}2966 \\
(6.65)\end{array}$ & $\begin{array}{l}3122.75 \\
(12.29)\end{array}$ & $\begin{array}{c}3167 \\
(13.88)\end{array}$ & $\begin{array}{l}3230.25 \\
(16.15)\end{array}$ & $\begin{array}{c}3437 \\
(23.59)\end{array}$ & 131.3750 & 3.9413 \\
\hline ta57 & $50 * 15$ & & 2943 & $\begin{array}{c}3101 \\
(5.37)\end{array}$ & $\begin{array}{l}3213.5 \\
(9.19)\end{array}$ & $\begin{array}{l}3256.5 \\
(10.65)\end{array}$ & $\begin{array}{l}3320.5 \\
(12.83)\end{array}$ & $\begin{array}{c}3485 \\
(18.42)\end{array}$ & 106.4220 & 27.6697 \\
\hline ta58 & $50 * 15$ & & 2885 & $\begin{array}{l}3103 \\
(7.56)\end{array}$ & $\begin{array}{l}3214.5 \\
(11.42)\end{array}$ & $\begin{array}{c}3273 \\
(13.45)\end{array}$ & $\begin{array}{l}3326.25 \\
(15.29)\end{array}$ & $\begin{array}{c}3438 \\
(19.17)\end{array}$ & 146.9060 & 104.3033 \\
\hline ta59 & $50 * 15$ & & 2655 & $\begin{array}{c}2940 \\
(10.73)\end{array}$ & $\begin{array}{r}3038.25 \\
(14.44)\end{array}$ & $\begin{array}{c}3090 \\
(16.38)\end{array}$ & $\begin{array}{l}3139.5 \\
(18.25)\end{array}$ & $\begin{array}{c}3294 \\
(24.07)\end{array}$ & 124.2970 & 119.3251 \\
\hline ta60 & $50 * 15$ & & 2723 & $\begin{array}{c}2921 \\
(7.27)\end{array}$ & $\begin{array}{l}3048.5 \\
(11.95)\end{array}$ & $\begin{array}{c}3104 \\
(13.99)\end{array}$ & $\begin{array}{c}3160 \\
(16.05)\end{array}$ & $\begin{array}{c}3339 \\
(22.62)\end{array}$ & 121.7970 & 86.4759 \\
\hline ta61 & $50 * 20$ & & 2868 & $\begin{array}{c}3258 \\
(13.60)\end{array}$ & $\begin{array}{l}3369.5 \\
(17.49)\end{array}$ & $\begin{array}{c}3424 \\
(19.39)\end{array}$ & $\begin{array}{c}3484 \\
(21.48)\end{array}$ & $\begin{array}{c}3649 \\
(27.23)\end{array}$ & 285.5000 & 216.9800 \\
\hline ta62 & $50 * 20$ & 2869 & 2872 & $\begin{array}{c}3306 \\
(15.11)\end{array}$ & $\begin{array}{c}3444 \\
(19.92)\end{array}$ & $\begin{array}{l}3493.5 \\
(21.64)\end{array}$ & $\begin{array}{c}3544 \\
(23.40)\end{array}$ & $\begin{array}{c}3730 \\
(29.87)\end{array}$ & 311.9850 & 293.2659 \\
\hline ta63 & $50 * 20$ & & 2755 & $\begin{array}{c}3130 \\
(13.61)\end{array}$ & $\begin{array}{c}3218.75 \\
(16.83)\end{array}$ & $\begin{array}{c}3273 \\
(18.80)\end{array}$ & $\begin{array}{l}3324.25 \\
(20.66)\end{array}$ & $\begin{array}{c}3487 \\
(26.57)\end{array}$ & 315.1250 & 302.5200 \\
\hline ta64 & $50 * 20$ & & 2702 & $\begin{array}{c}3008 \\
(11.32)\end{array}$ & $\begin{array}{l}3180.25 \\
(17.70)\end{array}$ & $\begin{array}{c}3230 \\
(19.54)\end{array}$ & $\begin{array}{l}3287.75 \\
(21.68)\end{array}$ & $\begin{array}{c}3431 \\
(26.98)\end{array}$ & 289.8440 & 153.6173 \\
\hline ta65 & $50 * 20$ & & 2725 & $\begin{array}{c}3104 \\
(13.91)\end{array}$ & $\begin{array}{l}3242.5 \\
(18.99)\end{array}$ & $\begin{array}{c}3286 \\
(20.59)\end{array}$ & $\begin{array}{c}3339 \\
(22.53)\end{array}$ & $\begin{array}{c}3569 \\
(30.97)\end{array}$ & 323.2340 & 42.0204 \\
\hline ta66 & $50 * 20$ & & 2845 & $\begin{array}{c}3198 \\
(12.41)\end{array}$ & $\begin{array}{c}3320 \\
(16.70)\end{array}$ & $\begin{array}{c}3361 \\
(18.14)\end{array}$ & $\begin{array}{l}3415.5 \\
(20.05)\end{array}$ & $\begin{array}{c}3585 \\
(26.01)\end{array}$ & 317.1570 & 63.4314 \\
\hline ta67 & $50 * 20$ & & 2825 & $\begin{array}{c}3209 \\
(13.59)\end{array}$ & $\begin{array}{c}3339 \\
(18.19)\end{array}$ & $\begin{array}{l}3389.5 \\
(19.98)\end{array}$ & $\begin{array}{l}3435.25 \\
(21.60)\end{array}$ & $\begin{array}{c}3578 \\
(26.65)\end{array}$ & 273.3440 & 46.4685 \\
\hline ta68 & $50 * 20$ & & 2784 & $\begin{array}{c}3133 \\
(12.54)\end{array}$ & $\begin{array}{c}3235.75 \\
(16.23)\end{array}$ & $\begin{array}{c}3283 \\
(17.92)\end{array}$ & $\begin{array}{l}3337.5 \\
(19.88)\end{array}$ & $\begin{array}{c}3542 \\
(27.23)\end{array}$ & 268.7810 & 223.0882 \\
\hline ta69 & $50 * 20$ & & 3071 & $\begin{array}{c}3366 \\
(9.61)\end{array}$ & $\begin{array}{l}3447.5 \\
(12.26)\end{array}$ & $\begin{array}{c}3517 \\
(14.52)\end{array}$ & $\begin{array}{c}3576 \\
(16.44)\end{array}$ & $\begin{array}{c}3739 \\
(21.75)\end{array}$ & 259.1720 & 33.6924 \\
\hline ta70 & $50 * 20$ & & 2995 & $\begin{array}{c}3449 \\
(15.16)\end{array}$ & $\begin{array}{c}3528.75 \\
(17.82)\end{array}$ & $\begin{array}{c}3582 \\
(19.60)\end{array}$ & $\begin{array}{r}3633.25 \\
(21.31)\end{array}$ & $\begin{array}{c}3815 \\
(27.38)\end{array}$ & 279.5150 & 58.6982 \\
\hline
\end{tabular}

The information of these tables can be visualised using boxplots. They show that the quality achieved is more dependent on the ratio $\mathrm{n} / \mathrm{m}$ than on the absolute 
numbers of jobs and machines. There is no big dispersion of the solution values achieved by the algorithm in the 100 runs executed, so we say the algorithm is steady. The number of times the algorithm achieves the best values reported is high enough, so these values are not considered outliers of the distribution of the results. On the other end, the worse values occur very seldom and are outliers for the majority of the instances.

GRASP_B\&B: \% from best UB

GRASP_B\&B: \% from best UB
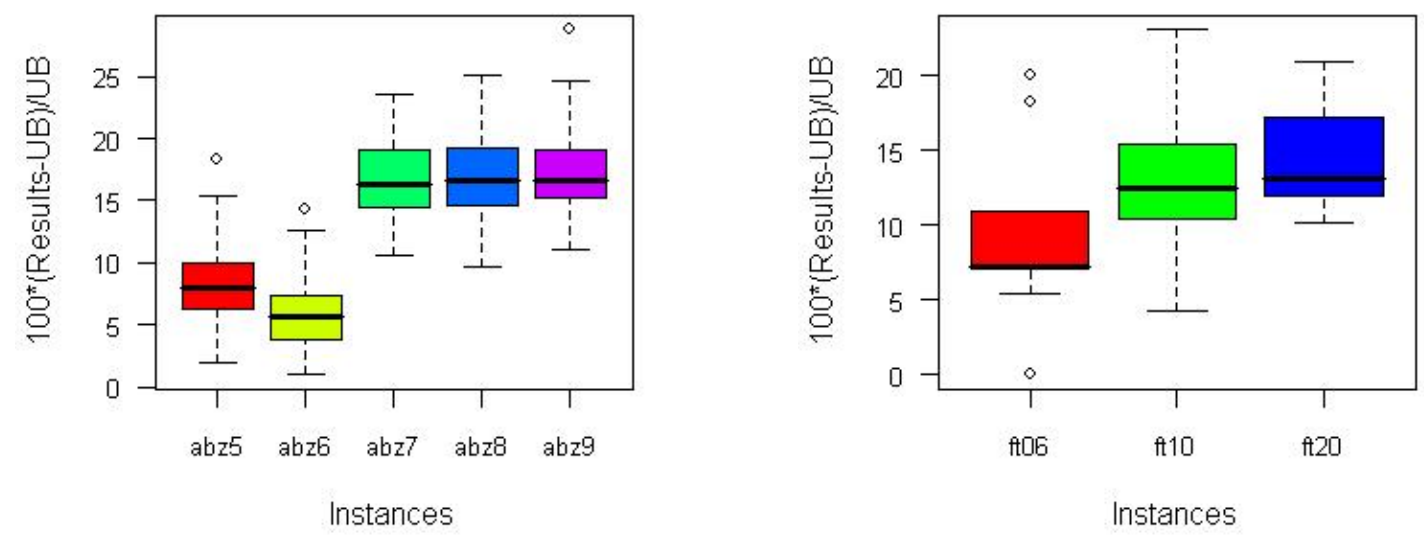

GRASP_B\&B: \% from best UB

GRASP_B\&B: \% from best UB
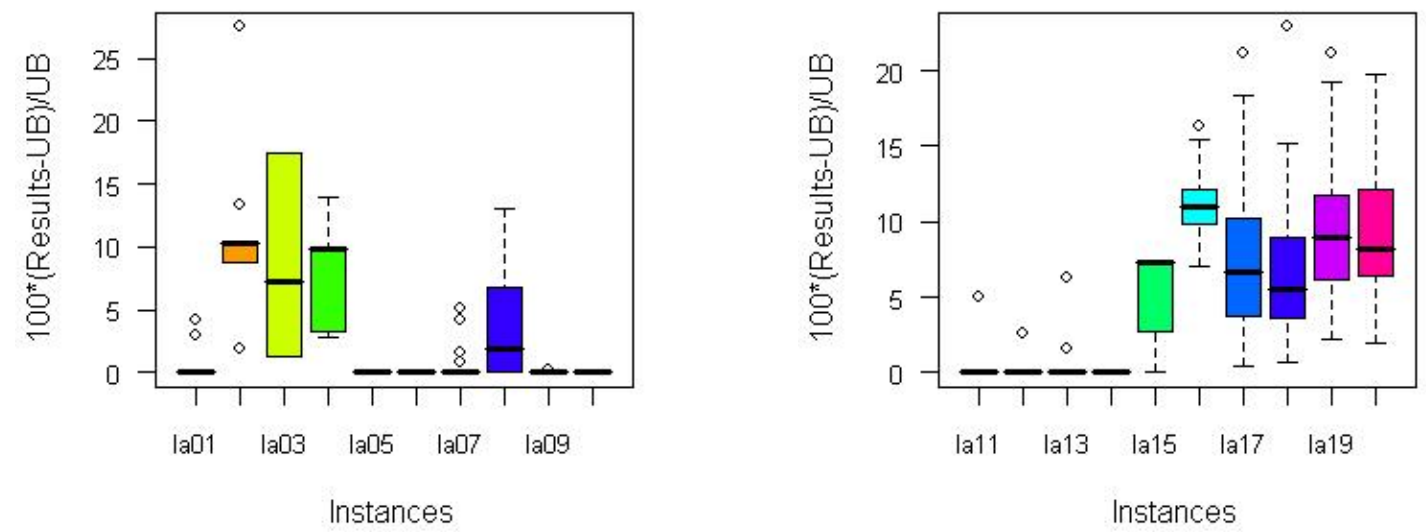
GRASP_B\&B: \% from best UB

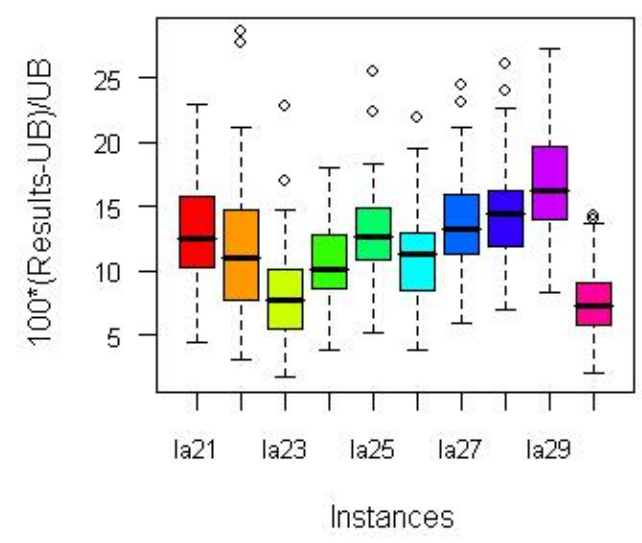

GRASP_B\&B: $\%$ from best UB

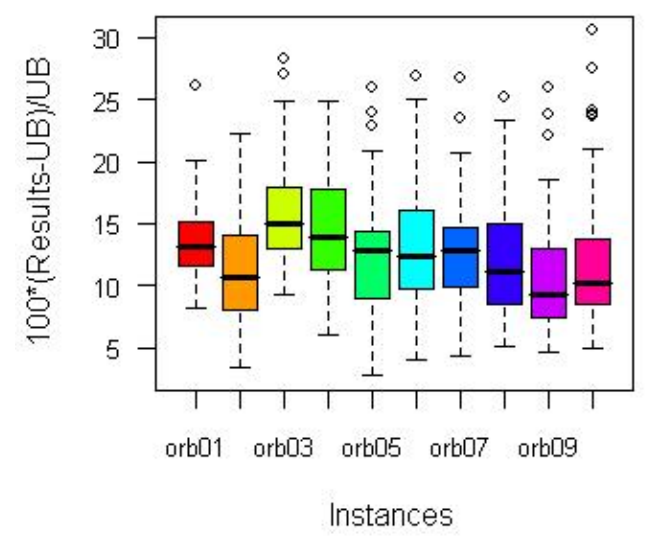

GRASP_B\&B: \% from best UB

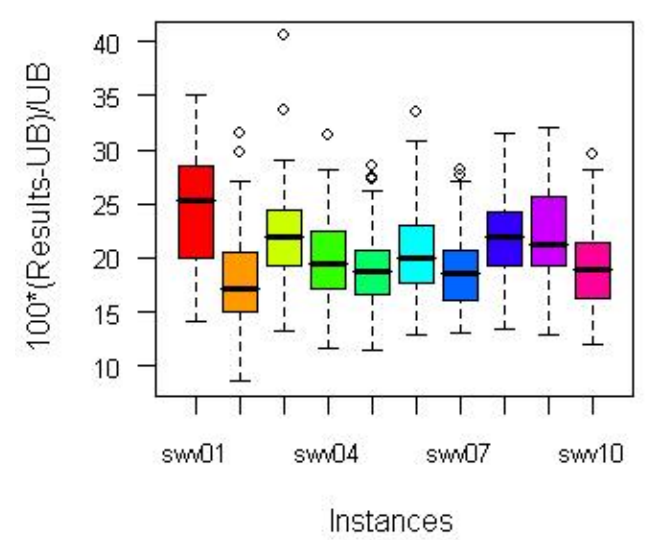

GRASP_B\&B: \% from best UB

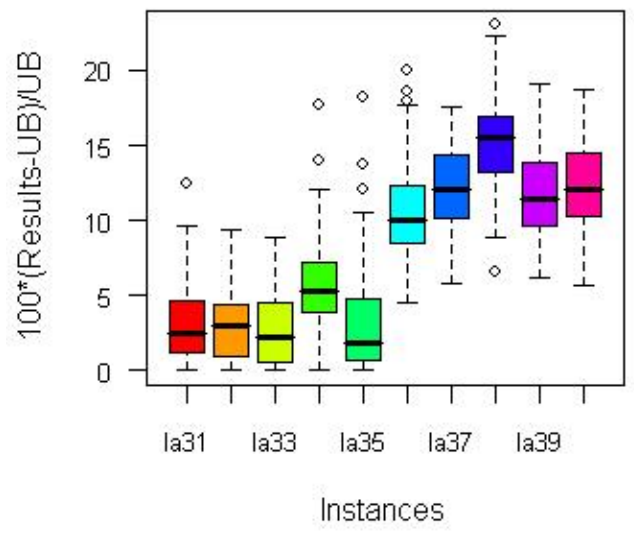

GRASP_B\&B: \% from best UB

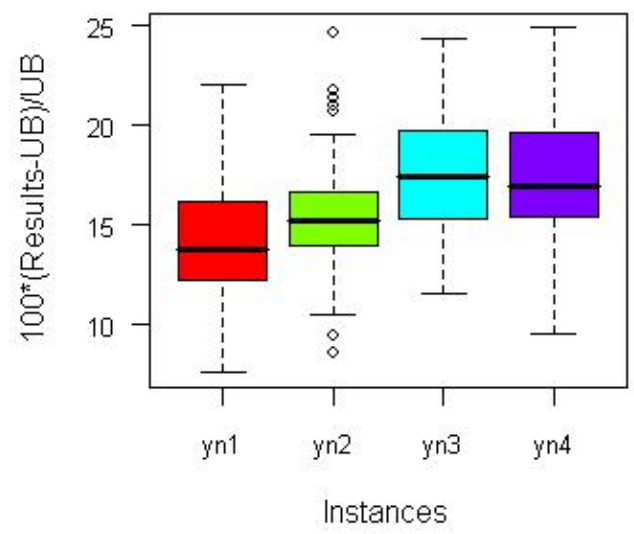

GRASP_B\&B: \% from best UB

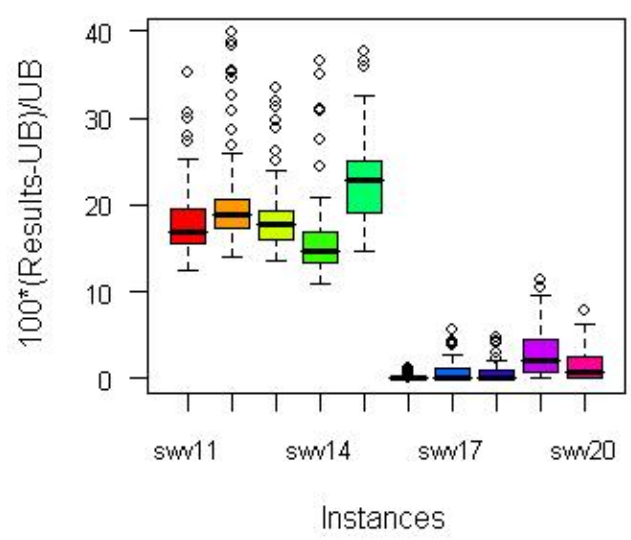


GRASP_B\&B: $\%$ from best UB

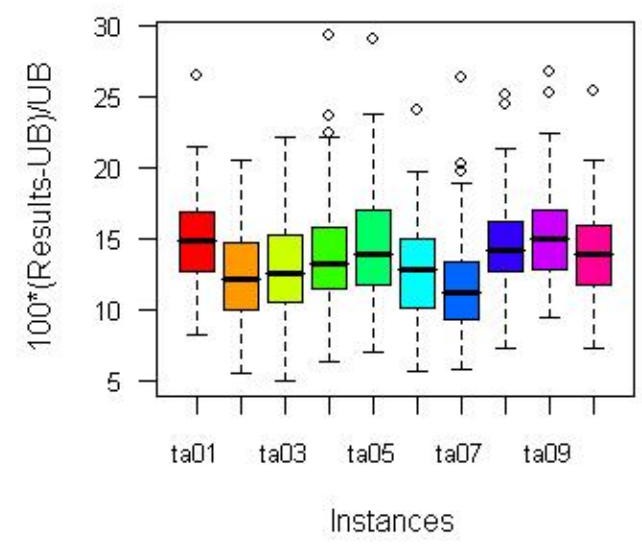

GRASP_B\&B: $\%$ from best UB

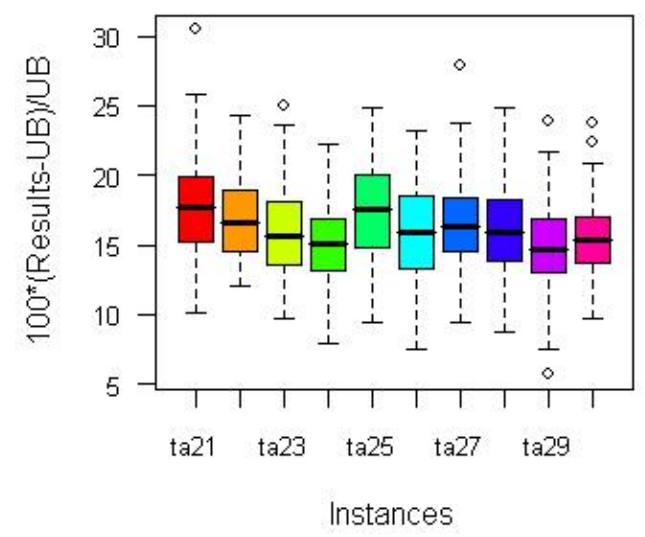

GRASP_B\&B: $\%$ from best UB

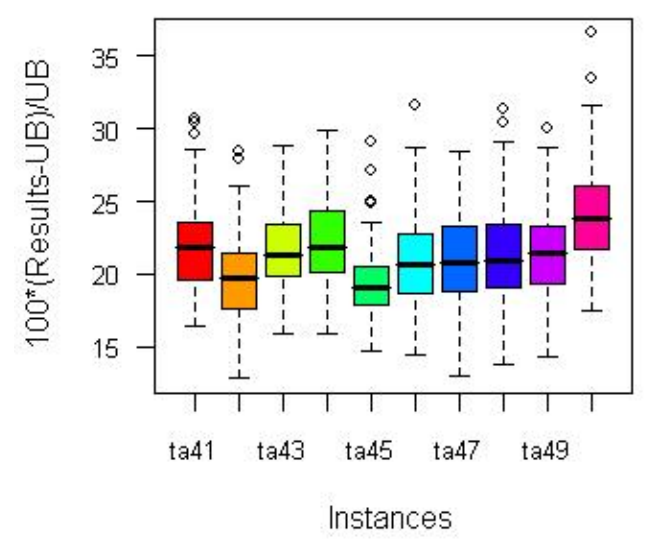

GRASP_B\&B: \% from best UB

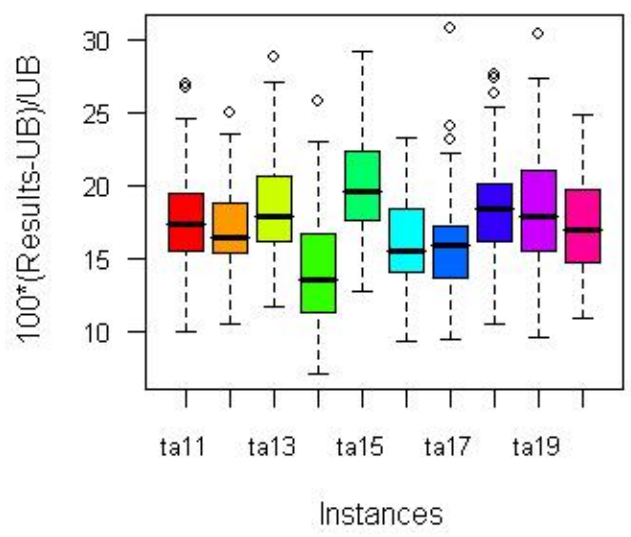

GRASP_B\&B: \% from best UB

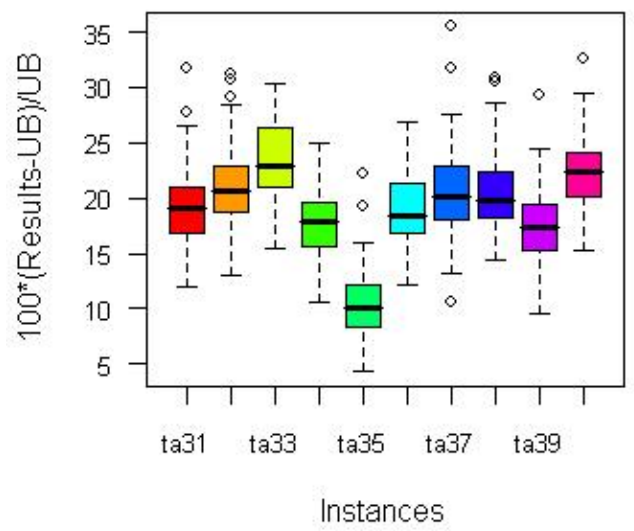

GRASP_B\&B: \% from best UB

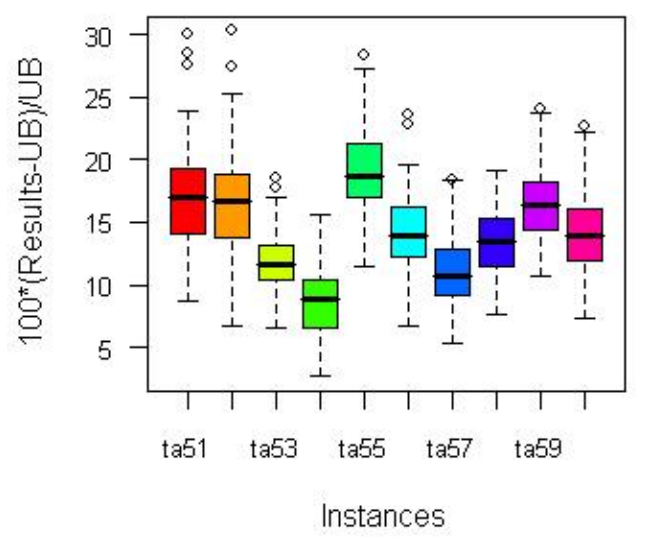


GRASP_B\&B: \% from best UB

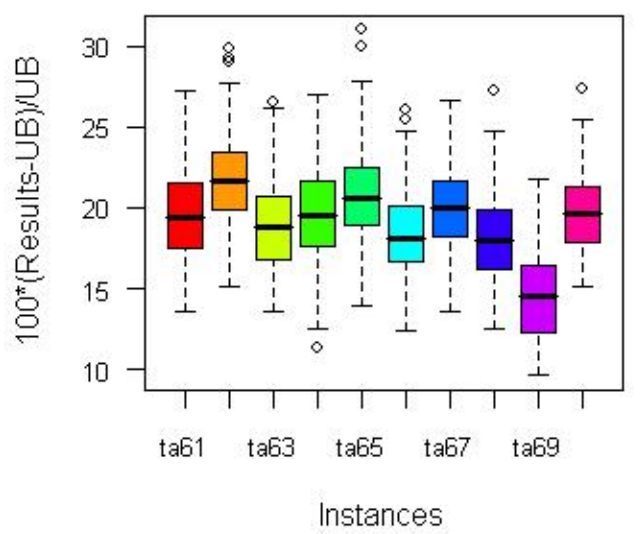

\subsection{Comparison to other procedures}

GRASP_B\&B OSH heuristic is a very simple GRASP algorithm with a construction phase very similar to the one of the shifting bottleneck. Therefore, we show comparative results to two other very similar methods: a simple GRASP heuristic of Binato et al (2001) and the Shifting Bottleneck heuristic by Adams et al (1988).

\subsubsection{Comparison to GRASP of Binato et al (2001)}

The GRASP heuristic by Binato et al (2001) has a different building step in the construction phase, which consists in scheduling one operation at each step. In their computational results, they present the time in seconds per thousand iterations (an iteration is one building phase followed by a local search) and the thousands of iterations. For a comparison purpose we multiply these values to get the total computation time. For GRASP_B\&B we present the total time of all runs (ttime), in seconds. As the tables show, our algorithm is much faster. Whenever our GRASP achieves a solution not worse than theirs, we present the respective value in bold. This happens for 26 of the 58 instances whose results where compared.

\begin{tabular}{|c|c|c|c|c|}
\hline name & GRASP_B\&B & ttime (s) & GRASP & time (s) \\
\hline abz5 & 1258 & 0.7650 & 1238 & 6030 \\
\hline abz6 & 952 & 0.7660 & 947 & 62310 \\
\hline abz7 & 725 & 10.9070 & 667 & 349740 \\
\hline abz8 & 734 & 10.5160 & 729 & 365820 \\
\hline
\end{tabular}




\begin{tabular}{|c|c|c|c|c|}
\hline abz9 & 754 & 10.4690 & 758 & 343710 \\
\hline name & GRASP_B\&B & ttime (s) & GRASP & time (s) \\
\hline $\mathrm{ft} 06$ & $\mathbf{5 5}$ & 0.1400 & 55 & 70 \\
\hline $\mathrm{ft} 10$ & 970 & 1.0000 & 938 & 261290 \\
\hline $\mathrm{ft} 20$ & 1283 & 0.4690 & 1169 & 387430 \\
\hline
\end{tabular}

\begin{tabular}{|c|c|c|c|c|}
\hline name & GRASP_B\&B & ttime (s) & GRASP & time (s) \\
\hline la01 & 666 & 0.1720 & 666 & 140 \\
\hline la02 & 667 & 0.1560 & 655 & 140 \\
\hline la03 & 605 & 0.2190 & 604 & 65130 \\
\hline la04 & 607 & 0.1710 & 590 & 130 \\
\hline la05 & 593 & 0.1100 & 593 & 130 \\
\hline la06 & 926 & 0.1710 & 926 & 240 \\
\hline la07 & 890 & 0.2030 & 890 & 250 \\
\hline la08 & 863 & 0.2970 & 863 & 240 \\
\hline la09 & 951 & 0.2810 & 951 & 290 \\
\hline la10 & 958 & 0.1410 & 958 & 250 \\
\hline la11 & 1222 & 0.2660 & 1222 & 410 \\
\hline la12 & 1039 & 0.2650 & 1039 & 390 \\
\hline la13 & 1150 & 0.3750 & 1150 & 430 \\
\hline la14 & 1292 & 0.2180 & 1292 & 390 \\
\hline la15 & 1207 & 0.9060 & 1207 & 410 \\
\hline la16 & 1012 & 0.7350 & 946 & 155310 \\
\hline la17 & 787 & 0.7660 & 784 & 60300 \\
\hline la18 & 854 & 0.7500 & 848 & 58290 \\
\hline la19 & 861 & 0.9690 & 842 & 31310 \\
\hline la20 & 920 & 0.8130 & 907 & 160320 \\
\hline la21 & 1092 & 2.0460 & 1091 & 325650 \\
\hline la22 & 955 & 1.7970 & 960 & 315630 \\
\hline la23 & 1049 & 1.8900 & 1032 & 65650 \\
\hline la24 & 971 & 1.8440 & 978 & 64640 \\
\hline la25 & 1027 & 1.7960 & 1028 & 64640 \\
\hline la26 & 1265 & 3.3750 & 1271 & 109080 \\
\hline la27 & 1308 & 3.5620 & 1320 & 110090 \\
\hline la28 & 1301 & 3.0000 & 1293 & 110090 \\
\hline la29 & 1248 & 3.2960 & 1293 & 112110 \\
\hline la30 & 1382 & 3.3280 & 1368 & 106050 \\
\hline la31 & 1784 & 7.0160 & 1784 & 231290 \\
\hline
\end{tabular}




\begin{tabular}{|c|c|c|c|c|}
\hline la32 & $\mathbf{1 8 5 0}$ & 6.2350 & 1850 & 241390 \\
\hline la33 & $\mathbf{1 7 1 9}$ & 7.9060 & 1719 & 241390 \\
\hline la34 & $\mathbf{1 7 2 1}$ & 8.2810 & 1753 & 240380 \\
\hline la35 & $\mathbf{1 8 8 8}$ & 5.6880 & 1888 & 222200 \\
\hline la36 & $\mathbf{1 3 2 5}$ & 4.2650 & 1334 & 115360 \\
\hline la37 & 1479 & 4.7970 & 1457 & 115360 \\
\hline la38 & 1274 & 5.1090 & 1267 & 115320 \\
\hline la39 & 1309 & 4.4530 & 1290 & 123200 \\
\hline la40 & 1291 & 5.3910 & 1259 & \\
\hline
\end{tabular}

\begin{tabular}{|c|c|c|c|c|}
\hline name & GRASP_B\&B & ttime (s) & GRASP & time (s) \\
\hline orb01 & 1145 & 0.9850 & 1070 & 116290 \\
\hline orb02 & 918 & 0.9530 & 889 & 152380 \\
\hline orb03 & 1098 & 1.0150 & 1021 & 124310 \\
\hline orb04 & 1066 & 1.1250 & 1031 & 124310 \\
\hline orb05 & 911 & 0.8750 & 891 & 112280 \\
\hline orb06 & 1050 & 1.0460 & 1013 & 124310 \\
\hline orb07 & 414 & 1.0630 & 397 & 128320 \\
\hline orb08 & 945 & 1.0310 & 909 & 124310 \\
\hline orb09 & 978 & 0.9060 & 945 & 124310 \\
\hline orb10 & 991 & 0.8430 & 953 & 116290 \\
\hline
\end{tabular}

\subsubsection{Comparison to the Shifting Bottleneck (Adams et al. 1988)}

The main difference of the Shifting Bottleneck procedure (Adams et al. 1988) and GRASP_B\&B is the random selection of the machine to be scheduled. In the Shifting Bottleneck the machine to be scheduled is always the bottleneck machine. The comparison between the shifting bottleneck procedure (Adams et al. 1988) and the GRASP_B\&B is also presented next. Comparing the computation times of both procedures, the GRASP_B\&B is slightly faster than the shifting bottleneck for smaller instances. Given the distinct computers used in the experiments we would say that this is not meaningful, but the difference does get accentuated as the dimensions grow. Whenever GRASP_B\&B achieves a solution better than the shifting bottleneck procedure, we present its value in bold. This happens in 29 of the 48 instances whose results where compared, and in 16 of the remaining 19 instances the best value found was the same. 


\begin{tabular}{|c|c|c|c|c|}
\hline name & GRASP_B\&B & ttime (s) & $\begin{array}{c}\text { Shifting } \\
\text { Bottleneck }\end{array}$ & time (s) \\
\hline abz5 & $\mathbf{1 2 5 8}$ & 0.7650 & 1306 & 5.7 \\
\hline abz6 & $\mathbf{9 5 2}$ & 0.7660 & 962 & 12.67 \\
\hline abz7 & $\mathbf{7 2 5}$ & 10.9070 & 730 & 118.87 \\
\hline abz8 & $\mathbf{7 3 4}$ & 10.5160 & 774 & 125.02 \\
\hline abz9 & 754 & 10.4690 & 751 & 94.32 \\
\hline
\end{tabular}

\begin{tabular}{|c|c|c|c|c|}
\hline name & GRASP_B\&B & ttime (s) & $\begin{array}{c}\text { Shifting } \\
\text { Bottleneck }\end{array}$ & time (s) \\
\hline $\mathrm{ft06}$ & 55 & 0.1400 & 55 & 1.5 \\
\hline $\mathrm{ft} 10$ & $\mathbf{9 7 0}$ & 1.0000 & 1015 & 10.1 \\
\hline $\mathrm{ft} 20$ & $\mathbf{1 2 8 3}$ & 0.4690 & 1290 & 3.5 \\
\hline
\end{tabular}

\begin{tabular}{|c|c|c|c|c|}
\hline name & GRASP_B\&B & ttime (s) & $\begin{array}{c}\text { Shifting } \\
\text { Bottleneck }\end{array}$ & time (s) \\
\hline la01 & 666 & 0.1720 & 666 & 1.26 \\
\hline la02 & 667 & 0.1560 & 720 & 1.69 \\
\hline la03 & 605 & 0.2190 & 623 & 2.46 \\
\hline la04 & 607 & 0.1710 & 597 & 2.79 \\
\hline la05 & 593 & 0.1100 & 593 & 0.52 \\
\hline la06 & 926 & 0.1710 & 926 & 1.28 \\
\hline la07 & 890 & 0.2030 & 890 & 1.51 \\
\hline la08 & 863 & 0.2970 & 868 & 2.41 \\
\hline la09 & $95^{\circ} 1$ & 0.2810 & 951 & 0.85 \\
\hline la10 & 958 & 0.1410 & 959 & 0.81 \\
\hline la11 & 1222 & 0.2660 & 1222 & 2.03 \\
\hline la12 & 1039 & 0.2650 & 1039 & 0.87 \\
\hline la13 & 1150 & 0.3750 & 1150 & 1.23 \\
\hline la14 & 1292 & 0.2180 & 1292 & 0.94 \\
\hline la15 & 1207 & 0.9060 & 1207 & 3.09 \\
\hline la16 & 1012 & 0.7350 & 1021 & 6.48 \\
\hline la17 & 787 & 0.7660 & 796 & 4.58 \\
\hline la18 & 854 & 0.7500 & 891 & 10.2 \\
\hline la19 & 861 & 0.9690 & 875 & 7.4 \\
\hline la20 & 920 & 0.8130 & 924 & 10.2 \\
\hline la21 & 1092 & 2.0460 & 1172 & 21.9 \\
\hline la22 & 955 & 1.7970 & 1040 & 19.2 \\
\hline la23 & 1049 & 1.8900 & 1061 & 24.6 \\
\hline
\end{tabular}




\begin{tabular}{|c|c|c|c|c|}
\hline la24 & $\mathbf{9 7 1}$ & 1.8440 & 1000 & 25.5 \\
\hline la25 & $\mathbf{1 0 2 7}$ & 1.7960 & 1048 & 27.9 \\
\hline la26 & $\mathbf{1 2 6 5}$ & 3.3750 & 1304 & 48.5 \\
\hline la27 & $\mathbf{1 3 0 8}$ & 3.5620 & 1325 & 45.5 \\
\hline la28 & 1301 & 3.0000 & 1256 & 28.5 \\
\hline la29 & $\mathbf{1 2 4 8}$ & 3.2960 & 1294 & 48 \\
\hline la30 & $\mathbf{1 3 8 2}$ & 3.3280 & 1403 & 37.8 \\
\hline la31 & 1784 & 7.0160 & 1784 & 38.3 \\
\hline la32 & 1850 & 6.2350 & 1850 & 29.1 \\
\hline la33 & 1719 & 7.9060 & 1719 & 25.6 \\
\hline la34 & 1721 & 8.2810 & 1721 & 27.6 \\
\hline la35 & 1888 & 5.6880 & 1888 & 21.3 \\
\hline la36 & $\mathbf{1 3 2 5}$ & 4.2650 & 1351 & 46.9 \\
\hline la37 & $\mathbf{1 4 7 9}$ & 4.7970 & 1485 & 6104 \\
\hline la38 & $\mathbf{1 2 7 4}$ & 5.1090 & 1280 & 57.5 \\
\hline la39 & $\mathbf{1 3 0 9}$ & 4.4530 & 1321 & 71.8 \\
\hline la40 & $\mathbf{1 2 9 1}$ & 5.3910 & 1326 & 76.7 \\
\hline
\end{tabular}

\section{Conclusions}

In this work we present a very simple Optimized Search Heuristic, the GRASP_B\&B to solve the Job-Shop Scheduling problem. This method is intended to be a starting point for a more elaborated metaheuristic, since it obtains reasonable solutions in very short running times. The main idea behind the GRASP_B\&B heuristic is to insert in each iteration of the building phase of the GRASP method the complete solution of one-machine scheduling problems solved by a branch-an-bound method, instead of insert one sequence of two individual operations as it is usual in other GRASP methods for this problem.

We have compared it with other similar methods also used as an initialization phase within more complex algorithms; namely a GRASP of Binato et. al (2001), which is the base for a GRASP with path-relinking procedure of Aiex et. al (2003), and the Shifting Bottleneck procedure of Adams et. al (1988), incorporated in the successful guided local search of Balas and Vazacopoulos (1991). The comparison to the GRASP of Binato et al (2001) shows that the GRASP_B\&B is much faster than theirs. The quality of their best solution is slightly better than ours in $60 \%$ of the instances tested. When comparing GRASP_B\&B with the Shifting Bottleneck, the first one is still faster, and it achieves better solutions, except for 3 of the comparable 
instances. Therefore we can conclude, that the GRASP_B\&B is a good method to use as the initialization phase of more elaborated and complex methods to solve the jobshop scheduling problem. As future research, we are working on this elaborated method also using OSH ideas, i.e. combing heuristic and exact methods procedures.

\section{Acknowledgement}

Susana Fernandes' work is suported by the the programm POCI2010 of the Portuguese Fundação para a Ciência e Tecnologia. Helena R. Lourenço’s work is supported by Ministerio de Educación y Ciencia, Spain, SEC2003-01991/ECO.

\section{References}

1 Adams, J., E. Balas and D. Zawack (1988). "The Shifting Bottleneck Procedure for Job Shop Scheduling." Management Science, vol. 34(3): pp. 391-401.

2 Applegate, D. and W. Cook (1991). "A Computational Study of the Job-Shop Scheduling Problem." ORSA Journal on Computing, vol. 3(2): pp. 149-156.

3 Balas, E. and A. Vazacopoulos (1998). "Guided Local Search with Shifting Bottleneck for Job Shop Scheduling." Management Science, vol. 44(2): pp. 262275.

4 Binato, S., W. J. Hery, D. M. Loewenstern and M. G. C. Resende (2001). "A GRASP for Job Shop Scheduling." In C.C. Ribeiro and P. Hansen, editors, Essays and surveys on metaheuristics, pp. 59-79. Kluwer Academic Publishers.

5 Carlier, J. (1982). "The one-machine sequencing problem." European Journal of Operational Research, vol. 11: pp. 42-47.

6 Caseau, Y. and F. Laburthe (1995), "Disjunctive scheduling with task intervals", Technical Report LIENS, 95-25, Ecole Normale Superieure Paris.

7 Chen, S., S. Talukdar and N. Sadeh (1993). "Job-shop-scheduling by a team of asynchronous agentes", Proceedings of the IJCAI-93 Workshop on KnowledgeBased Production, Scheduling and Control. Chambery France.

8 Danna, E., E. Rothberg and C. L. Pape (2005). "Exploring relaxation induced neighborhoods to improve MIP solutions." Mathematical Programming, Ser. A, vol. 102: pp. 71-90.

9 Dell'Amico, M. and M. Trubian (1993). "Applying Tabu-Search to the Job-Shop Scheduling Problem." 
10 Denzinger, J. and T. Offermann (1999). "On Cooperation between Evolutionary Algorithms and other Search Paradigms", Proceedings of the 1999 Congress on Evolutionary Computational.

11 Feo, T. and M. Resende (1995). "Greedy Randomized Adaptive Search Procedures." Journal of Global Optimization, vol. 6: pp. 109-133.

12 Fernandes, S. and H.R. Lourenço (2007), "Optimized Search Heuristics", Universitat Pompeu Fabra, Barcelona, Spain.

(http://www.econ.upf.edu/ ramalhin/OSHwebpage/index.html)

13 Fisher, H. and G. L. Thompson (1963). Probabilistic learning combinations of local job-shop scheduling rules. In J. F. Muth and G. L. Thompson eds. Industrial Scheduling. pp. 225-251. Prentice Hall, Englewood Cliffs.

14 Garey, M. R. and D. S. Johnson (1979). Computers and Intractability: A Guide to the Theory of NP-Completeness. San Francisco, Freeman.

15 Jain, A. S. and S. Meeran (1999). "Deterministic job shop scheduling: Past, present and future." European Journal of Operational Research, vol. 133: pp. 390-434.

16 Lawrence, S. (1984), "Resource Constrained Project Scheduling: an Experimental Investigation of Heuristic Scheduling techniques", Graduate School of Industrial Administration, Carnegie-Mellon University.

17 Lourenço, H. R. (1995). "Job-shop scheduling: Computational study of local search and large-step optimization methods." European Journal of Operational Research, vol. 83: pp. 347-367.

18 Lourenço, H. R. and M. Zwijnenburg (1996). Combining large-step optimization with tabu-search: Application to the job-shop scheduling problem. In I. H. Osman and J. P. Kelly eds. Meta-heuristics: Theory \& Applications. Kluwer Academic Publishers.

19 Nowicki, E. and C. Smutnicki (2002), "Some new tools to solve the job shop problem", Technical Report, 60/2002, Institute of Engineering Cybernetics, Wroclaw University of Technology.

20 Nowicki, E. and C. Smutnicki (2005). "An Advanced Tabu Search Algorithm for the Job Shop Problem." Journal of Scheduling, vol. 8: pp. 145-159.

21 Nowicki, E. and C. Smutniki (1996). "A Fast Taboo Search Algorithm for the Job Shop Problem." Management Science, vol. 42(6): pp. 797-813.

22 Roy, B. and B. Sussman (1964), "Les probèms d'ordonnancement avec constraintes disjonctives", Note DS 9 bis, SEMA, Paris. 
23 Schaal, A., A. Fadil, H. M. Silti and P. Tolla (1999). "Meta heuristics diversification of generalized job shop scheduling based upon mathematical programming techniques", Proceedings of the Cp-ai-or'99.

24 Schrage, L. (1970). "Solving resource-constrained network problems by implicit enumeration: Non pre-emptive case." Operations Research, vol. 18: pp. 263-278.

25 Storer, R. H., S. D. Wu and R. Vaccari (1992). "New search spaces for sequencing problems with application to job shop scheduling." Management Science, vol. 38(10): pp. 1495-1509.

26 Taillard, E. D. (1993). "Benchmarks for Basic Scheduling Problems." European Journal of Operational Research, vol. 64(2): pp. 278-285.

27 Taillard, É. D. (1994). "Parallel Taboo Search Techniques for the Job Shop Scheduling Problem." ORSA Journal on Computing, vol. 6(2): pp. 108-117.

28 Tamura, H., A. Hirahara, I. Hatono and M. Umano (1994). "An approximate solution method for combinatorial optimisation." Transactions of the Society of Instrument and Control Engineers, vol. 130: pp. 329-336.

29 Yamada, T. and R. Nakano (1992). A genetic algorithm applicable to large-scale job-shop problems. In R. Manner and B. Manderick eds. Parallel Problem Solving from Nature 2. pp. 281-290. Elsevier Science. 